\title{
Simplest little Higgs model revisited: Hidden mass relation, unitarity, and naturalness
}

\author{
Kingman Cheung, ${ }^{1,4,5, *}$ Shi-Ping He, ${ }^{2, \dagger}$ Ying-nan Mao, ${ }^{3, *}$ Chen Zhang, ${ }^{1, \S}$ and Yang Zhou ${ }^{2, \|}$ \\ ${ }^{1}$ Physics Division, National Center for Theoretical Sciences, Hsinchu 300, Taiwan \\ ${ }^{2}$ Institute of Theoretical Physics and State Key Laboratory of Nuclear Physics and Technology, \\ Peking University, Beijing 100871, China \\ ${ }^{3}$ Center for Future High Energy Physics and Theoretical Physics Division, \\ Institute of High Energy Physics, Chinese Academy of Sciences, Beijing 100049, China \\ ${ }^{4}$ Department of Physics, National Tsing Hua University, Hsinchu 300, Taiwan \\ ${ }^{5}$ Division of Quantum Phases and Devices, School of Physics, Konkuk University, \\ Seoul 143-701, Republic of Korea
}

(Received 20 March 2018; published 4 June 2018)

\begin{abstract}
We analyze the scalar potential of the simplest little Higgs (SLH) model in an approach consistent with the spirit of continuum effective field theory (CEFT). By requiring correct electroweak symmetry breaking (EWSB) with the $125 \mathrm{GeV}$ Higgs boson, we are able to derive a relation between the pseudoaxion mass $m_{\eta}$ and the heavy top mass $m_{T}$, which serves as a crucial test of the SLH mechanism. By requiring $m_{\eta}^{2}>0$ an upper bound on $m_{T}$ can be obtained for any fixed SLH global symmetry breaking scale $f$. We also point out that an absolute upper bound on $f$ can be obtained by imposing partial wave unitarity constraint, which in turn leads to absolute upper bounds of $m_{T} \lesssim 19 \mathrm{TeV}, m_{\eta} \lesssim 1.5 \mathrm{TeV}$, and $m_{Z^{\prime}} \lesssim 48 \mathrm{TeV}$. We present the allowed region in the three-dimensional parameter space characterized by $f, t_{\beta}, m_{T}$, taking into account the requirement of valid EWSB and the constraint from perturbative unitarity. We also propose a strategy of analyzing the fine-tuning problem consistent with the spirit of CEFT and apply it to the SLH. We suggest that the scalar potential and fine-tuning analysis strategies adopted here should also be applicable to a wide class of little Higgs and twin Higgs models, which may reveal interesting relations as crucial tests of the related EWSB mechanism and provide a new perspective on assessing their degree of fine-tuning.
\end{abstract}

DOI: 10.1103/PhysRevD.97.115001

\section{INTRODUCTION}

The discovery of the $125 \mathrm{GeV}$ Higgs-like particle $[1,2]$ is undoubtedly a great success of the standard model (SM) in which the electroweak symmetry breaking (EWSB) is achieved via the nonzero vacuum expectation value associated with a single $S U(2)_{L}$ doublet Higgs field. Nevertheless, there is no a priori reason to believe that the EWSB must be realized in the minimal manner dictated by the SM. There are in fact compelling signs that physics beyond the SM (BSM) should exist to account for issues like dark matter, neutrino mass and oscillation, baryon

\footnotetext{
*cheung@phys.nthu.edu.tw

†sphe@pku.edu.cn

*maoyn@ihep.ac.cn

§czhang@cts.nthu.edu.tw

"yangzhou9103@gmail.com
}

Published by the American Physical Society under the terms of the Creative Commons Attribution 4.0 International license. Further distribution of this work must maintain attribution to the author(s) and the published article's title, journal citation, and DOI. Funded by SCOAP ${ }^{3}$. asymmetry of the universe, etc. In a general setting, the new physics responsible for the explanation of these issues would interact with the SM Higgs field such that the Higgs mass becomes radiatively unstable. ${ }^{1}$ It is therefore preferable that some mechanism should exist to stabilize the Higgs mass. Very often these stabilizing mechanisms would require modification of the minimal EWSB mechanism realized by one single $S U(2)_{L}$ doublet Higgs field. Also, such modification should be related to a scale not much higher than the electroweak scale so that the stabilizing mechanism itself does not introduce a severe fine-tuning problem.

One popular candidate of such stabilizing mechanisms is weak scale supersymmetry (SUSY), which is representative of weakly-coupled extensions of the SM. Compared to scenarios which invoke strong dynamics, SUSY extensions

\footnotetext{
${ }^{1}$ This is the Higgs mass naturalness or fine-tuning problem, which we refer the reader to Refs. [3,4] and references therein for representative discussion in literature. In this work we do not distinguish semantically between "naturalness problem" and "fine-tuning problem" of the Higgs mass.
} 
are more predictive in terms of calculability. On the other hand, SUSY entails the introduction of superpartners for every SM particle, and hence nearly a doubling of degrees of freedom in the theory. None of these superpartners have been observed so far. Also, a large number of new parameters associated with these new degrees of freedom are introduced, making the model quite complicated. It is therefore very desirable if there are other weakly-coupled ${ }^{2}$ theories which could stabilize the Higgs mass and at the same time require less degrees of freedom with simpler theoretical construction.

One interesting model building option consistent with this line of thinking is to use the little Higgs mechanism [5-8]. ${ }^{3}$ The essential ingredient of the little Higgs mechanism is collective symmetry breaking (CSB). In CSB, the Higgs boson is realized as a Nambu-Goldstone boson (NGB) of some global symmetry breaking. However, the global symmetry is also explicitly broken in such a manner that at least two operators are needed at the same time to break enough symmetry so that the Higgs ceases to be an exact NGB. Because more operators are needed to renormalize the Higgs mass, the radiative stability of the theory is improved. Enlargement of gauge group is generally required for the implementation of the little Higgs mechanism. One of the simplest possibilities is the simplest little Higgs (SLH) model [11,12], in which the electroweak gauge group is enlarged to $S U(3)_{L} \times U(1)_{X}$. Accordingly, two scalar triplets are needed, the vacuum expectation values of which leads to the following spontaneous global symmetry breaking pattern:

$$
\begin{aligned}
& {\left[S U(3)_{1} \times U(1)_{1}\right] \times\left[S U(3)_{2} \times U(1)_{2}\right]} \\
& \quad \rightarrow\left[S U(2)_{1} \times U(1)_{1}\right] \times\left[S U(2)_{2} \times U(1)_{2}\right]
\end{aligned}
$$

The global symmetry is also explicitly broken by gauge and Yukawa interactions, but in a collective manner to improve the radiative stability of the model. The $125 \mathrm{GeV}$ Higgs boson is supposed to be one of the pseudo-Nambu-Goldstone bosons of the global symmetry breaking in Eq. (1). The particle content is quite economical. Especially, for the scalar sector, with the radial modes integrated out, there is only one physical degree of freedom left (usually referred to as the "pseudo-axion" [13,14]) besides the $125 \mathrm{GeV}$ Higgs boson.

When it comes to the extraction of EWSB predictions in the SLH, there are two approaches adopted in the literature. ${ }^{4}$ The first approach is to calculate under the assumption of "no large direct contribution to the scalar potential from the physics at the cutoff" $[12,15]$, which will

\footnotetext{
${ }^{2}$ Here for an effective theory, being "weakly-coupled" is supposed to hold in its range of validity.

${ }^{3}$ For early reviews, see Refs. [9,10].

${ }^{4}$ These two approaches are not peculiar to the study of the SLH. They have been widely adopted for many little Higgs and twin Higgs models as well.
}

be abbreviated as the "NDCC assumption" in the rest of the paper. In this approach, the tree-level scalar potential is assumed to vanish (except for a technically natural $\mu$ term which gives mass to the pseudoaxion). The one-loop scalar effective potential is generated by Yukawa and gauge interactions, triggering EWSB and making the Higgs boson massive. The divergent loop momentum integral is assumed to be cut off at the naive dimensional analysis (NDA) [16] cutoff of the associated nonlinear sigma model. The second approach is to simply abandon the NDCC assumption and treat the associated model parameters as effectively free parameters [17].

Both approaches mentioned above have significant drawbacks if we attempt to derive quantitative predictions from the SLH. In the first approach, the regularization cutoff encountered in one-loop effective potential calculations is invested with a physical meaning, rather than being treated via the standard renormalization procedure discussed in quantum field theory (QFT) textbooks [18] and the original Coleman-Weinberg paper [19]. ${ }^{5}$ The practice of imparting a physical meaning to the regularization cutoff could be somewhat misleading on certain occasions [22]. Therefore it is always desirable that the relevant problems be treated in a more rigorous and solid manner with a clear conceptual foundation. Moreover, if we stick to the first kind of approach (with its conceptual foundation put aside for the moment), we would have difficulty in determining the cutoff value to be used. In the SLH literature like Ref. [12], a cutoff value of $4 \pi f_{1}$ is used where $f_{1}$ denotes the smaller one of the vacuum expectation values of the two scalar triplets. This value comes from the NDA which only gives a qualitative rather than quantitative estimate of the scale up to which the nonlinear sigma model is expected to be valid. If we consider the requirement of perturbative unitarity, then usually the cutoff value is much smaller than the NDA estimate [23]. Therefore the results obtained by plugging in any specific cutoff value cannot be taken too seriously and we would have no idea about the associated uncertainties. A further objection is that there seems to be no a priori reason to believe that there is no large direct contribution from the physics at the cutoff, and explanations are needed to clarify what is meant exactly by "large."

\footnotetext{
${ }^{5}$ We note that there might be an interpretational ambiguity about the first approach. One could choose not to interpret this approach as imparting physical meaning to the regularization cutoff without renormalization. Instead, one might interpret the cutoff (usually denoted as $\Lambda$ ) as the renormalization scale [20]. However, many papers (e.g., $[8,12,21]$ ) retain a $\Lambda^{2}$ term in the Coleman-Weinberg potential, and demonstrate the cancellation of quadratic divergence by showing its coefficient vanishes in the considered model. This is at least formally in conflict with the interpretation of $\Lambda$ as a renormalization scale in a mass-independent renormalization scheme. We will discuss this alternative interpretation further in Secs. III and IV.
} 
In the second approach, as adopted in Ref. [17], although the ad hoc assumption used in the first approach is abandoned, the parameters related to the EWSB in the SLH are all treated free parameters which can vary independently. Therefore, the predictivity of the SLH in terms of its EWSB is lost to a large extent. As we will show in the following sections, even if we allow direct contribution to the scalar potential from the physics at the cutoff, there is still an important mass relation which connects various parameters of the model dictated by the requirement of correct EWSB with the $125 \mathrm{GeV}$ Higgs boson.

In this paper we would argue that it is both possible and preferable to adopt an approach consistent with the spirit of continuum effective field theory (CEFT) [24], which leads to clear and calculable predictions regarding the EWSB in the SLH. In the CEFT approach, the UV cutoff only appears in the regularization and should be removed after renormalization. This is in contrast with the Wilsonian effective field theory in which there is indeed an intrinsic UV cutoff associated with the theory, and is awkward to be employed for ordinary phenomenological studies.

With the above in mind, we perform an analysis of the SLH scalar potential in the CEFT approach. We explicitly write down the scalar quartic term required by the renormalization procedure without any assumption on the contribution from the physics at the cutoff. This does not make the EWSB prediction in the SLH completely arbitrary because the renormalization is constrained by the symmetry of theory. Minimization of the scalar effective potential up to one-loop level is supposed to yield an electroweak vacuum expectation value and a Higgs mass consistent with experiments. As we will show, these requirements lead to an interesting mass relation between the pseudoaxion mass $m_{\eta}$ and the heavy top mass $m_{T}$, which serves as a crucial test of the SLH mechanism. Due to the anticorrelation between $m_{\eta}$ and $m_{T}$, requiring $m_{\eta}^{2}>0$ leads to an upper bound on $m_{T}$ for any given SLH global symmetry breaking scale $f \equiv \sqrt{f_{1}^{2}+f_{2}^{2}}$, where $f_{1}, f_{2}$ denote the vacuum expectation values of the two scalar triplets before EWSB, respectively. Another prediction of the EWSB in the SLH is that the minimal value of the ratio between the two scalar triplets $t_{\beta} \equiv \frac{f_{2}}{f_{1}}$ (assuming $f_{2} \geq f_{1}$ for the moment) is expected to increase with the increase of $f$. We note that the heavy gauge boson masses in the SLH is mainly determined by the overall scale $f$ while the NDA/unitarity cutoff is supposed to be determined by the smaller one of the two scalar vacuum expectation values. This implies that a too large $f$ will push the heavy gauge boson masses into a region where perturbation theory might not be reliable. In this work we require all particle masses in the low energy theory do not exceed the perturbative unitarity bound derived for the SLH nonlinear sigma fields. Consequently, we are able to obtain absolute upper bounds on the scale $f$ and all the relevant particle masses in the theory with which a self-contained EFT for SLH below its unitarity cutoff can be established.

A further advantage of the CEFT approach is that it automatically offers a clear and convenient framework for a quantitative investigation of the naturalness problem. The physical content of a quantum field theory can be formulated independent of the regularization cutoff and the naturalness problem should be formulated using only physical, renormalized quantities. The real issue of the naturalness problem is radiative stability. We note that in CEFT, the radiative structure of the theory is embodied in its renormalization group equations (RGE) in a massindependent renormalization scheme, keeping in mind that when going below heavy particle thresholds, we need to put in by hand the Appelquist-Carazzone decoupling [25] by integrating out heavy degrees of freedom and shifting to a low energy EFT suitable for the description of the low energy phenomena [24]. Within this picture, two sources of fine-tuning can be easily identified: one is related to the RGE evolution between thresholds, and the other is related to the matching onto a low energy EFT when crossing thresholds. Based on these considerations, we explicitly calculate these two kinds of fine-tuning for the case of the SLH, using results obtained from our scalar potential analysis. Furthermore, it is possible to combine the two fine-tuning measures and obtain a total fine-tuning for the SLH which is a measure of how sensitive the electroweak scale parameters are to the variation of the parameters defined at the unitarity cutoff of the theory. We also clarify the connection between our fine-tuning definition and some conventionally adopted fine-tuning definitions used for the SLH in the literature.

The paper is organized as follows. In Sec. II, we introduce the basic setup of the SLH model, including the field content and the Lagrangian. Section III analyzes the SLH scalar potential in an approach consistent with the spirit of CEFT with the deriviation of the $m_{\eta}-m_{T}$ mass relation and characterization of the allowed parameter space consistent with valid EWSB and unitarity. Section IV presents a quantitative analysis of the degree of fine-tuning in the SLH based on the CEFT picture. In Sec. V we present our discussion and conclusion.

\section{THE SIMPLEST LITTLE HIGGS}

In the SLH, the electroweak gauge group is enlarged to $S U(3)_{L} \times U(1)_{X}$. Two scalar triplets $\Phi_{1}, \Phi_{2}$ are introduced as nonlinear sigma fields and parametrized in the following manner to realize the spontaneous global symmetry breaking pattern in Eq. (1)

$$
\Phi_{1}=\exp \left(\frac{i \Theta^{\prime}}{f}\right) \exp \left(\frac{i t_{\beta} \Theta}{f}\right)\left(\begin{array}{c}
0 \\
0 \\
f c_{\beta}
\end{array}\right)
$$




$$
\Phi_{2}=\exp \left(\frac{i \Theta^{\prime}}{f}\right) \exp \left(-\frac{i \Theta}{f t_{\beta}}\right)\left(\begin{array}{c}
0 \\
0 \\
f s_{\beta}
\end{array}\right) .
$$

Here we have introduced the shorthand notation $s_{\beta} \equiv \sin \beta, c_{\beta} \equiv \cos \beta, t_{\beta} \equiv \tan \beta$. The Goldstone decay constant $f$ is supposed to be at least a few TeV. $\Theta$ and $\Theta^{\prime}$ are $3 \times 3$ matrix fields, parametrized as

$$
\Theta=\frac{\eta}{\sqrt{2}}+\left(\begin{array}{cc}
\boldsymbol{0}_{2 \times 2} & h \\
h^{\dagger} & 0
\end{array}\right), \quad \Theta^{\prime}=\frac{\zeta}{\sqrt{2}}+\left(\begin{array}{cc}
\mathbf{0}_{2 \times 2} & k \\
k^{\dagger} & 0
\end{array}\right)
$$

where $\eta$ is the physical pseudoaxion discussed in literature $[13,14]$, and $h$ and $k$ are parametrized as ( $v$ denotes the vacuum expectation value of the Higgs doublet)

$$
\begin{gathered}
h=\left(\begin{array}{c}
h^{0} \\
h^{-}
\end{array}\right), \quad h^{0}=\frac{1}{\sqrt{2}}(v+H-i \chi) \\
k=\left(\begin{array}{c}
k^{0} \\
k^{-}
\end{array}\right), \quad k^{0}=\frac{1}{\sqrt{2}}(\sigma-i \omega) .
\end{gathered}
$$

For future convenience, we introduce the notation

$$
\hat{h} \equiv\left(h^{\dagger} h\right)^{1 / 2} .
$$

Some remarks about the above parametrization are in order. First, there is considerable freedom in parametrizing the scalar triplets $\Phi_{1}$ and $\Phi_{2}$. For instance, in Eqs. (2) and (3) we have adopted a double exponential parametrization. Also, in Eq. (4) we use the identity matrix as the generator for the $\eta, \zeta$ fields. It is certainly legitimate to use instead a single exponential parametrization, and/or some other appropriate matrix like $T^{8} \equiv \frac{\lambda^{8}}{2}\left(\lambda^{8}\right.$ denotes the eighth GellMann matrix) for the $\eta$, $\zeta$ generator. These different parametrizations are mathematically related by field redefinition and are thus physically equivalent. Nevertheless, using the identity matrix as the generator for $\eta, \zeta$ fields simplifies the calculation, and as pointed out in Ref. [26], the double exponential parametrization does not induce mixing of $\eta$ with unphysical Goldstones in the term responsible for $\eta$ mass. Second, we have assumed that among various Goldstone components, only the real part of $h^{0}$ may acquire a nonzero vacuum expectation value. Especially, the $\eta$ field has zero vacuum expectation value and therefore $C P$ is not spontaneously broken.(We refer the reader to Ref. [27] for a previous paper on the phenomenology of the spontaneous $C P$-violating SLH.) Such a vacuum configuration can be obtained by minimization of the scalar effective potential, as will be demonstrated in Sec. III.

In the SLH, under the full gauge group $S U(3)_{C} \times$ $S U(3)_{L} \times U(1)_{X}, \quad \Phi_{1}$ and $\Phi_{2}$ have quantum number $(\mathbf{1}, \mathbf{3})_{-\frac{1}{3}}$. The gauge kinetic term of $\Phi_{1}$ and $\Phi_{2}$ can thus be written as

$$
\mathcal{L}_{g k}=\left(D_{\mu} \Phi_{1}\right)^{\dagger}\left(D^{\mu} \Phi_{1}\right)+\left(D_{\mu} \Phi_{2}\right)^{\dagger}\left(D^{\mu} \Phi_{2}\right)
$$

in which the covariant derivative can be expressed as

$$
D_{\mu}=\partial_{\mu}-i g A_{\mu}^{a} T^{a}+i g_{x} Q_{x} B_{\mu}^{x}, \quad g_{x}=\frac{g t_{W}}{\sqrt{1-t_{W}^{2} / 3}}
$$

In the above equation, $A_{\mu}^{a}$ and $B_{\mu}^{x}$ denote $S U(3)_{L}$ and $U(1)_{X}$ gauge fields, respectively. $g$ and $g_{x}$ denote the coupling constants of $S U(3)_{L}$ and $U(1)_{X}$ gauge groups, respectively. It is convenient to trade $g_{x}$ for $t_{W} \equiv \tan \theta_{W}$ for future derivation. $T^{a}=\frac{\lambda^{a}}{2}$ where $\lambda^{a}, a=1, \ldots, 8$ denote the GellMann matrices. For $\Phi_{1}, \Phi_{2}, Q_{x}=-\frac{1}{3}$. Following Ref. [28], we parametrize the $S U(3)_{L}$ gauge bosons as

$$
\begin{aligned}
A_{\mu}^{a} T^{a}= & \frac{A_{\mu}^{3}}{2}\left(\begin{array}{ccc}
1 & 0 & 0 \\
0 & -1 & 0 \\
0 & 0 & 0
\end{array}\right)+\frac{A_{\mu}^{8}}{2 \sqrt{3}}\left(\begin{array}{ccc}
1 & 0 & 0 \\
0 & 1 & 0 \\
0 & 0 & -2
\end{array}\right) \\
& +\frac{1}{\sqrt{2}}\left(\begin{array}{ccc}
0 & W_{\mu}^{+} & Y_{\mu}^{0} \\
W_{\mu}^{-} & 0 & X_{\mu}^{-} \\
Y_{\mu}^{0 \dagger} & X_{\mu}^{+} & 0
\end{array}\right)
\end{aligned}
$$

with the first-order neutral gauge boson mixing relation $\left(c_{W} \equiv \cos \theta_{W}, s_{W} \equiv \sin \theta_{W}\right)$

$$
\left(\begin{array}{c}
A^{3} \\
A^{8} \\
B_{x}
\end{array}\right)=\left(\begin{array}{ccc}
0 & c_{W} & -s_{W} \\
\sqrt{1-\frac{t_{W}^{2}}{3}} & \frac{s_{W} t_{W}}{\sqrt{3}} & \frac{s_{W}}{\sqrt{3}} \\
-\frac{t_{W}}{\sqrt{3}} & s_{W} \sqrt{1-\frac{t_{W}^{2}}{3}} & c_{W} \sqrt{1-\frac{t_{W}^{2}}{3}}
\end{array}\right)\left(\begin{array}{c}
Z^{\prime} \\
Z \\
A
\end{array}\right) .
$$

We note in passing that in the presence of vacuum misalignment (i.e., $v \neq 0$ ), generally speaking $\eta, \zeta, \chi, \omega$ will not be canonically normalized. Also, there could exist "unexpected" vector-scalar mixing terms such as $Z_{\mu} \partial^{\mu} \eta$ at tree level. This kind of situation is not uncommon for models which invoke gauged nonlinear sigma fields. Even if $\zeta, \chi, \omega$ can be rotated away by gauge transformations, terms like $Z_{\mu} \partial^{\mu} \eta$ certainly cannot be eliminated by a naive gauge rotation. A systematic procedure for diagonalizing such a vector-scalar system in gauge theories, including the elimination of "unexpected" vector-scalar two-point mixing via an appropriate gauge-fixing, is outlined in Ref. [26] and applied to the SLH. The implication is that a further transformation among the $\eta, \zeta, \chi, \omega$ has to be made to derive the correct masses and couplings related to these particles. For the main purpose of the present paper, this subtlety will only lead to a $\mathcal{O}\left(v^{2} / f^{2}\right)$-suppressed correction to the derived $\eta$ mass.

We now turn to the Yukawa Lagrangian. Since the electroweak gauge group is now $S U(3)_{L} \times U(1)_{X}$, new 
fermions need to be introduced to furnish complete representations of the gauge group. This can be done elegantly in an anomaly-free manner [12,29,30] which we adopt here. In the lepton Yukawa sector, the SM lefthanded lepton doublets are enlarged to $S U(3)_{L}$ triplets $L_{m}=\left(\nu_{L}, \ell_{L}, i N_{L}\right)_{m}^{T}$ with $Q_{x}=-\frac{1}{3}(m=1,2,3$ is the family index). There are also right-handed singlet lepton fields $\ell_{R m}$ with $Q_{x}=-1$ and $N_{R m}$ with $Q_{x}=0$. The lepton Yukawa Lagrangian can be written as [28]

$\mathcal{L}_{L Y}=i \lambda_{N}^{m} \bar{N}_{R m} \Phi_{2}^{\dagger} L_{m}+\frac{i \lambda_{\ell}^{m n}}{\Lambda} \bar{\ell}_{R m} \epsilon_{i j k} \Phi_{1}^{i} \Phi_{2}^{j} L_{n}^{k}+$ H.c.

The charged leptons $e, \mu, \tau$ pick up their masses through the dimension-five operators in $\mathcal{L}_{L Y}$, in which an energy scale $\Lambda$ is introduced to make the $3 \times 3$ mass matrix $\lambda_{\ell}$ dimensionless. The dimension-four operators in $\mathcal{L}_{L Y}$ makes the new leptons $N_{R}$ 's massive. It should be noted that light neutrinos $\nu_{L}$ 's remain massless with $\mathcal{L}_{L Y}$, although their masses can be straightforwardly included by adding $\left(\Phi_{2}^{\dagger} L\right)^{2}$ operators.

The anomaly-free requirement leads to the following quark-field content

$$
\begin{array}{lrrrr}
Q_{1}=\left(d_{L},-u_{L}, i D_{L}\right)^{T}, & d_{R}, & u_{R}, & D_{R} \\
Q_{2}=\left(s_{L},-c_{L}, i S_{L}\right)^{T}, & s_{R}, & c_{R}, & S_{R} \\
Q_{3}=\left(t_{L}, b_{L}, i T_{L}\right)^{T}, & t_{R}, & b_{R}, & T_{R}
\end{array}
$$

Here $Q_{1}, Q_{2}$ transform under $\overline{\mathbf{3}}$ representation of $S U(3)_{L}$ with $Q_{x}=0 . Q_{3}$ transforms under 3 representation of $S U(3)_{L}$ with $Q_{x}=\frac{1}{3}$. The right-handed quark fields are all $S U(3)_{L}$ singlets with various $U(1)_{X}$ charges. More specifically, $u_{R}, c_{R}, t_{R}, T_{R}$ carry $Q_{x}=\frac{2}{3}$ while $d_{R}, s_{R}, b_{R}, D_{R}$, $S_{R}$ carry $Q_{x}=-\frac{1}{3}$. The quark Yukawa Lagrangian can be written as [28]

$$
\begin{aligned}
\mathcal{L}_{Q Y}= & i \lambda_{1}^{t} \bar{u}_{R 3}^{1} \Phi_{1}^{\dagger} Q_{3}+i \lambda_{2}^{t} \bar{u}_{R 3}^{2} \Phi_{2}^{\dagger} Q_{3}+i \frac{\lambda_{b}^{m}}{\Lambda} \bar{d}_{R m} \epsilon_{i j k} \Phi_{1}^{i} \Phi_{2}^{j} Q_{3}^{k} \\
& +i \lambda_{1}^{d n} \bar{d}_{R n}^{1} Q_{n}^{T} \Phi_{1}+i \lambda_{2}^{d n} \bar{d}_{R n}^{2} Q_{n}^{T} \Phi_{2} \\
& +i \frac{\lambda_{u}^{m n}}{\Lambda} \bar{u}_{R m} \epsilon_{i j k} \Phi_{1}^{* i} \Phi_{2}^{* j} Q_{n}^{k}+\text { H.c. }
\end{aligned}
$$

In the above equation, $n=1,2$ is the family index for the first two generations of quark triplets. $d_{R m}$ runs over $\left(d_{R}, s_{R}, b_{R}, D_{R}, S_{R}\right)$ and $u_{R m}$ runs over $\left(u_{R}, c_{R}, t_{R}, T_{R}\right)$. $u_{R 3}^{1}, u_{R 3}^{2}$ are linear combinations of $t_{R}$ and $T_{R} . d_{R n}^{1}, d_{R n}^{2}$ are linear combinations of $d_{R}$ and $D_{R}$ for $n=1$ and of $s_{R}$ and $S_{R}$ for $n=2$.

The CSB mechanism in the SLH deserves comment. In the bosonic sector, $\mathcal{L}_{g k}$ automatically realizes CSB, while in the fermionic sector, we have deliberately chosen the dimension-four operators in $\mathcal{L}_{L Y}, \mathcal{L}_{Q Y}$ to ensure CSB.
Especially, in Eq. (12) we do not write down a $\bar{N}_{R} \Phi_{1}^{\dagger} L$ term which is allowed by gauge symmetry but formally violates CSB when $\bar{N}_{R} \Phi_{2}^{\dagger} L$ is also present. In Eq. (16), the crucial ingredient for scalar potential analysis is the top sector Lagrangian

$$
\mathcal{L}_{t Y}=i \lambda_{1}^{t} \bar{u}_{R 3}^{1} \Phi_{1}^{\dagger} Q_{3}+i \lambda_{2}^{t} \bar{u}_{R 3}^{2} \Phi_{2}^{\dagger} Q_{3} \subset \mathcal{L}_{Q Y}
$$

in which the CSB is manifest. The dimension-five operators in Eqs. (12) and (16) actually violate CSB. Nevertheless, these sources of violation are proportional to light fermion Yukawa and their effect on scalar potential analysis can be safely neglected.

If in Eq. (12) the $i \lambda_{N}^{m} \bar{N}_{R m} \Phi_{2}^{\dagger} L_{m}$ term is neglected at the moment, then we could restrict the range of $t_{\beta}$ to be $t_{\beta} \geq 1$ without loss of generality. This is because in this case we are always free to label the scalar triplet with smaller vacuum expectation value as $\Phi_{1}$. However, when we require the very presence of $i \lambda_{N}^{m} \bar{N}_{R m} \Phi_{2}^{\dagger} L_{m}$ term in Eq. (12), this labeling redundancy does not hold any more, and we need to consider both $t_{\beta} \geq 1$ and $t_{\beta}<1$. Nevertheless, for the analysis in the present paper, it is found that the labeling redundancy $t_{\beta} \leftrightarrow \frac{1}{t_{\beta}}$ still holds to a good approximation since the correction only comes in at $\mathcal{O}\left(v^{2} / f^{2}\right)$ in the input parameter. Therefore, in the rest of this paper we will still present the results by focusing on the $t_{\beta} \geq 1$ case.

\section{HIDDEN MASS RELATION FROM SCALAR POTENTIAL ANALYSIS}

\section{A. Scalar potential in the SLH}

Up to now, we have not described the scalar potential in the SLH yet. In Sec. I, we mentioned two approaches commonly adopted in the literature. In one of them, the relevant model parameters are treated effectively as free parameters and the predictivity is lost to a large extent. Therefore, let us scrutinize the other approach (used in Refs. [12,15]) to see whether there is room for improvement.

In the approach adopted by Refs. [12,15], the tree-level scalar potential is assumed to vanish except for the $\mu$ term

$$
\mathcal{L}_{\mu}=\mu^{2}\left(\Phi_{1}^{\dagger} \Phi_{2}+\text { H.c. }\right) \text {. }
$$

The Yukawa and gauge interactions then generate a potential at one-loop level, triggering EWSB. At one-loop, the effective-potential calculation contains logarithmic UV divergence due to fermion and gauge boson loops. In Refs. [12,15], this logarithmic divergence is treated by imposing a momentum cutoff $\Lambda$, which is taken to be $4 \pi f_{1}$ in Ref. [12] and $4 \pi f$ in Ref. [15].

From a CEFT point of view, the appearance of UV divergence in the calculation signals the need for 
renormalization. A similar but simpler example is scalar quantum electrodynamics (QED). If we do not write down a scalar quartic term at tree level, then when doing one-loop calculation of scalar scattering processes we will still encounter UV divergence which needs to be absorbed by adding counterterms. From the viewpoint of renormalization theory, a consistent approach is to introduce in the bare Lagrangian a scalar quartic term. Calculation at oneloop order can then be done via renormalized perturbation theory, in which the bare Lagrangian is split into the renormalized part and the counterterm part. The UV divergences encountered in loop calculations can then be absorbed by counterterms in appropriate renormalization schemes. The renormalization procedure will usually introduce an unphysical scale (renormalization scale) into calculation. Requiring physical quantities to be independent of this unphysical scale leads to the notion of running couplings as a consequence of solving the relevant CallanSymanzik equation.

Nevertheless, in the literature, a crude momentum cutoff $\Lambda$ is imposed, and there is an ambiguity concerning the interpretation of $\Lambda$. In the Appendix of Ref. [12], as can be inferred from the constants appearing in the expression of Coleman-Weinberg $(\mathrm{CW})$ potential and the text, the CW potential expression corresponds to $\overline{\mathrm{DR}}$ scheme [31,32] rather than a sharp momentum cutoff regularization without renormalization. In Ref. [20], $\overline{\mathrm{MS}}$ scheme is used and $\Lambda=$ $4 \pi f_{1}$ is interpreted as the renormalization scale. However, if $\Lambda$ is regarded as the renormalization scale, then the choice of its value should be arbitrary in principle since physical predictions should be renormalization group invariant. This seems to contradict the fact that the EWSB predictions in the previous literature indeed rely on say, setting $\Lambda=4 \pi f_{1}$. As will be discussed in Sec. IV, the contradiction disappears only if we are forced to accept the assumption of vanishing contribution to the scalar potential from the physics at the cutoff. In spite of this, interpreting $\Lambda$ as a renormalization scale is still in conflict with the fact that in Refs. [12,20] (and many other papers) a $\Lambda^{2}$ term is sometimes retained to discuss cancellation of quadratic divergence or related issues.

In the present paper we opt for an approach consistent with the spirit of CEFT. We first write down the bare scalar potential $V_{B}$ as follows

$$
V_{B}=-\mu_{B}^{2}\left(\Phi_{1 B}^{\dagger} \Phi_{2 B}+\Phi_{2 B}^{\dagger} \Phi_{1 B}\right)+\lambda_{B}\left|\Phi_{1 B}^{\dagger} \Phi_{2 B}\right|^{2} .
$$

The subscript " $B$ " denotes bare quantities. In $V_{B}$ we retain operators up to dimension-four and assume the effects of higher-dimensional operators can be neglected (which is not an inconsistent power-counting assumption). We note that both operators in Eq. (19) violate CSB. However, this violation is not really harmful. For the $\mu$ term, if we neglect the small light fermion Yukawa, it softly breaks the global $U(1)$ symmetry in which two scalar triplets undergo opposite phase rotations. [The pseudoaxion $\eta$ actually corresponds to the pseudo-Goldstone of this spontaneously broken $U(1)$.] The scalar quartic coupling $\lambda_{B}$ is a dimensionless parameter and will not induce a serious fine-tuning problem unless its renormalized counterpart takes some extreme value (this issue will be discussed in more detail in Sec. IV). We emphasize that the inclusion of the scalar quartic operator $\left|\Phi_{1 B}^{\dagger} \Phi_{2 B}\right|^{2}$ is required by renormalization. It provides the necessary counterterm to absorb the UV divergence encountered in the calculation of radiative correction to the scalar effective potential. A dimensionfour operator $\left(\Phi_{1 B}^{\dagger} \Phi_{2 B}\right)^{2}+$ H.c. is also allowed by gauge symmetry, however it would formally cause a hard breaking of the global $U(1)$ symmetry that protects the $\mu$ term. Therefore we do not include it in $V_{B}$.

We now move on to the calculation of scalar effective potential via renormalized perturbation theory. The treelevel effective potential is now written as

$$
V_{\text {tree }}=-\mu^{2}\left(\Phi_{1}^{\dagger} \Phi_{2}+\Phi_{2}^{\dagger} \Phi_{1}\right)+\lambda_{R}\left|\Phi_{1}^{\dagger} \Phi_{2}\right|^{2} .
$$

In the above equation all quantities (couplings, fields) are renormalized ones, with $\Phi_{1}, \Phi_{2}$ assuming the parametrization used in Eqs. (2) and (3). At one-loop level, we take into account the contribution from gauge interaction and top sector Yukawa, and express the scalar potential at small field value (i.e., $\hat{h} \ll f$ ) as

$$
V_{1-\text { loop }}=V_{1 \text {-loop }}^{\mathrm{s}}+V_{1 \text {-loop }}^{\mathrm{ns}}
$$

in which the $S U(3)$-symmetric part $V_{1-\text { loop }}^{\mathrm{s}}$ and $S U(3)$ nonsymmetric part $V_{1-\text { loop }}^{\text {ns }}$ are respectively given by

$$
\begin{gathered}
V_{1 \text {-loop }}^{\mathrm{s}}=\bar{\lambda}\left|\Phi_{1}^{\dagger} \Phi_{2}\right|^{2} \\
V_{1 \text {-loop }}^{\mathrm{ns}}=\Delta(\hat{h}) \hat{h}^{4} .
\end{gathered}
$$

Here the coefficient of the $\hat{h}^{4}$ term is written as $\Delta(\hat{h})$, indicating that it is field-dependent. We therefore call $\Delta(\hat{h})$ a field form factor, emphasizing it is not a field-independent constant. Combining the tree-level and one-loop contributions, the scalar effective potential $V$ (defined as the sum of $V_{\text {tree }}$ and $\left.V_{\text {1-loop }}\right)$ is given by

$$
V=-\mu^{2}\left(\Phi_{1}^{\dagger} \Phi_{2}+\Phi_{2}^{\dagger} \Phi_{1}\right)+\lambda\left|\Phi_{1}^{\dagger} \Phi_{2}\right|^{2}+\Delta(\hat{h}) \hat{h}^{4}
$$

in which

$$
\lambda \equiv \lambda_{R}+\bar{\lambda}
$$

In our treatment the counterterm contribution is included in $V_{1 \text {-loop }}$, therefore $\mu^{2}, \lambda, \Delta(\hat{h})$ in Eq. (24) are all finite quantities with no dependence on the regularization cutoff. 
Taking into account gauge boson and top sector Yukawa contributions, $\bar{\lambda}$ and $\Delta(\hat{h})$ are computed to be (Landau gauge and $\overline{\mathrm{MS}}$ scheme are adopted)

$$
\begin{gathered}
\bar{\lambda}=-\frac{3}{8 \pi^{2}}\left[\lambda_{t}^{2} \frac{M_{T}^{2}}{f^{2}}\left(\ln \frac{M_{T}^{2}}{\mu_{R}^{2}}-1\right)-\frac{1}{4} g^{2} \frac{M_{X}^{2}}{f^{2}}\left(\ln \frac{M_{X}^{2}}{\mu_{R}^{2}}-\frac{1}{3}\right)\right. \\
\left.-\frac{1}{8} g^{2}\left(1+t_{W}^{2}\right) \frac{M_{Z^{\prime}}^{2}}{f^{2}}\left(\ln \frac{M_{Z^{\prime}}^{2}}{\mu_{R}^{2}}-\frac{1}{3}\right)\right] \\
\Delta(\hat{h})=\frac{3}{16 \pi^{2}}\left\{\lambda_{t}^{4}\left[\ln \frac{M_{T}^{2}}{m_{t}^{2}(\hat{h})}-\frac{1}{2}\right]-\frac{1}{8} g^{4}\left[\ln \frac{M_{X}^{2}}{m_{W}^{2}(\hat{h})}-\frac{1}{2}\right]\right. \\
\left.-\frac{1}{16} g^{4}\left(1+t_{W}^{2}\right)^{2}\left[\ln \frac{M_{Z^{\prime}}^{2}}{m_{Z}^{2}(\hat{h})}-\frac{1}{2}\right]\right\} .
\end{gathered}
$$

In the above equations, $\mu_{R}$ is the renormalization scale in the $\overline{\mathrm{MS}}$ scheme. We deliberately avoid the use of $\Lambda$ here to prevent any interpretational ambiguity. $\lambda_{t}$ is defined as

$$
\lambda_{t} \equiv \frac{\lambda_{1}^{t} \lambda_{2}^{t}}{\sqrt{\lambda_{1}^{t 2} c_{\beta}^{2}+\lambda_{2}^{t 2} s_{\beta}^{2}}}
$$

where $\lambda_{1}^{t}, \lambda_{2}^{t}$ are the two Yukawa couplings in the top sector, introduced in Eq. (16). $M_{T}^{2}, M_{X}^{2}, M_{Z^{\prime}}^{2}$ are defined as

$$
\begin{gathered}
M_{T}^{2} \equiv\left(\lambda_{1}^{t 2} c_{\beta}^{2}+\lambda_{2}^{t 2} s_{\beta}^{2}\right) f^{2} \\
M_{X}^{2} \equiv \frac{1}{2} g^{2} f^{2} \\
M_{Z^{\prime}}^{2} \equiv \frac{2}{3-t_{W}^{2}} g^{2} f^{2} .
\end{gathered}
$$

They are related to physical mass squared of the relevant particles as follows

$$
\begin{gathered}
M_{T}^{2}=m_{T}^{2}+m_{t}^{2} \\
M_{X}^{2}=m_{X}^{2}+m_{W}^{2} \\
M_{Z^{\prime}}^{2}=m_{Z^{\prime}}^{2}+m_{Z}^{2}
\end{gathered}
$$

in which $m_{T}, m_{t}$ denote the physical mass of the heavy top $T$ and the top quark $t, m_{X}, m_{W}$ denote the physical mass of the $X$ boson and $W$ boson, $m_{Z^{\prime}}, m_{Z}$ denote the physical mass of the $Z^{\prime}$ boson and $Z$ boson, respectively. We distinguish between $M_{T}$ and $m_{T}, M_{X}$ and $m_{X}, M_{Z^{\prime}}$ and $m_{Z^{\prime}}$, although the numerical differences are very small. $m_{t}^{2}(\hat{h}), m_{W}^{2}(\hat{h}), m_{Z}^{2}(\hat{h})$ are field-dependent mass squared, which we use the following leading order expression in the field form factor

$$
m_{t}^{2}(\hat{h})=\lambda_{t}^{2} \hat{h}^{2}
$$

$$
\begin{gathered}
m_{W}^{2}(\hat{h})=\frac{1}{2} g^{2} \hat{h}^{2} \\
m_{Z}^{2}(\hat{h})=\frac{1}{2} g^{2}\left(1+t_{W}^{2}\right) \hat{h}^{2} .
\end{gathered}
$$

We will see that retaining the field-dependence in these expressions is important for the quantitative study of $m_{\eta}-m_{T}$ correlation.

We note that in our calculation of $V$, the gauge boson and fermionic contributions are considered at one-loop order, while the contribution from the scalar sector itself is only considered to tree level. This is consistent since the leading contribution of gauge boson and fermion fields to the scalar effective potential arise at one-loop order while the leading contribution of scalar fields to the scalar effective potential arises already at tree level in our treatment. As long as perturbation theory is valid, the scalar one-loop contribution should always be small compared to the scalar tree level contribution which we already take into account.

\section{B. Analysis of the scalar effective potential}

Having obtained the scalar effective potential, Eq. (24), with the expression of $\bar{\lambda}$ and $\Delta(\hat{h})$ given in Eqs. (26) and (27), we now begin to analyze its physical implications. It is helpful to first pin down the dimension of parameter space that we are dealing with. For the purpose of scalar potential analysis, if we consider $m_{t}, g$ and $t_{W}$ as known and fixed, then before fixing $v$ and the $C P$-even Higgs mass (denoted as $m_{h}$ ), we have five adjustable parameters that enter into $V$. For instance, we may choose the five parameters to be $f, t_{\beta}, M_{T}, \mu, \lambda$. Once these five parameters are given, other quantities like $v, m_{h}$, and $\eta$ mass $m_{\eta}$ can be derived. Alternatively, we can utilize the measured value of electroweak vacuum expectation value and $C P$-even Higgs mass to eliminate two of the five initial parameters (say, $\mu$ and $\lambda$ ), leaving the remaining three $f, t_{\beta}, M_{T}$ as independent parameters to characterize the parameter space. The $\eta$ mass, which is associated with a second derivative of $V$ at its local minimum, can be determined from $f, t_{\beta}, M_{T}$. This is exactly the hidden mass relation that we wish to point out in the present paper $\left(M_{T}\right.$ is related to $m_{T}$ via Eq. (32). This relation can be viewed as a $m_{\eta}-m_{T}$ mass relation given $f$ and $t_{\beta}$, and serve as a crucial test of the SLH mechanism. This relation can also be viewed as a $m_{\eta}-m_{h}$ mass relation. When we consider only tree-level scalar effective potential, an $m_{\eta}-m_{h}$ mass relation can be obtained which is valid for any value of the Lagrangian parameters. When one-loop gauge boson and fermion contributions are taken into account, the correction to $m_{\eta}-m_{h}$ mass relation is then automatically finite and becomes a calculable prediction of the symmetry structure of the theory. In this sense, this hidden mass relation is a zeroth-order natural relation [18], which we now begin to derive. 
A convenient starting point is the scalar effective potential $V$ in Eq. (24) at small field value. With the parametrization Eqs. (2) and (3), $\Phi_{1}^{\dagger} \Phi_{2}$ can be expressed in terms of $\hat{h}$ and $\eta$ exactly as follows [33]

$\Phi_{1}^{\dagger} \Phi_{2}=f^{2} s_{\beta} c_{\beta} \exp \left(-\frac{i}{\sqrt{2} f s_{\beta} c_{\beta}} \eta\right) \cos \left(\frac{1}{f s_{\beta} c_{\beta}} \hat{h}\right)$.

Making use of this expression we may express $V$ in Eq. (24) as a function of two real variables $\hat{h}$ and $\eta$

$$
\begin{aligned}
V= & -2 \mu^{2} f^{2} s_{\beta} c_{\beta} \cos \left(\frac{1}{\sqrt{2} f s_{\beta} c_{\beta}} \eta\right) \cos \left(\frac{1}{f s_{\beta} c_{\beta}} \hat{h}\right) \\
& +\lambda f^{4} s_{\beta}^{2} c_{\beta}^{2} \cos ^{2}\left(\frac{1}{f s_{\beta} c_{\beta}} \hat{h}\right)+\Delta \hat{h}^{4} .
\end{aligned}
$$

Here and in the following, we simply use $\Delta$ to represent the field form factor $\Delta(\hat{h})$, keeping in mind its field-dependence. The electroweak vacuum should correspond to a stationary point of $V$, satisfying $\frac{\partial V}{\partial \eta}=\frac{\partial V}{\partial \hat{h}}=0$, i.e.,

$$
\begin{array}{r}
\sqrt{2} \mu^{2} f \sin \left(\frac{1}{\sqrt{2} f s_{\beta} c_{\beta}} \eta\right) \cos \left(\frac{1}{f s_{\beta} c_{\beta}} \hat{h}\right)=0 \\
2 \mu^{2} f \cos \left(\frac{1}{\sqrt{2} f s_{\beta} c_{\beta}} \eta\right) \sin \left(\frac{1}{f s_{\beta} c_{\beta}} \hat{h}\right) \\
-\lambda f^{3} s_{\beta} c_{\beta} \sin \left(\frac{2}{f s_{\beta} c_{\beta}} \hat{h}\right)+4 \Delta \hat{h}^{3}+\Delta^{\prime} \hat{h}^{4}=0
\end{array}
$$

in which

$$
\Delta^{\prime} \equiv \frac{d \Delta}{d \hat{h}}
$$

We are concerned with the case of no spontaneous $C P$ violation, i.e., $\eta=0$. In this case, Eq. (40) is automatically satisfied, and Eq. (41) becomes

$$
\begin{aligned}
& 2 \mu^{2} f \sin \left(\frac{1}{f s_{\beta} c_{\beta}} \hat{h}\right)-\lambda f^{3} s_{\beta} c_{\beta} \sin \left(\frac{2}{f s_{\beta} c_{\beta}} \hat{h}\right) \\
& +4 \Delta \hat{h}^{3}+\Delta^{\prime} \hat{h}^{4}=0 .
\end{aligned}
$$

Suppose $P_{0}=\left(0, \hat{h}_{0}\right)$ corresponds to the $(\eta, \hat{h})$ field configuration of the electroweak vacuum and therefore $\hat{h}_{0}$ is a solution of Eq. (43). According to Eq. (4) $\hat{h}_{0}$ is related to $v$ by

$$
\hat{h}_{0}=\frac{v}{\sqrt{2}} .
$$

The elements of the Hessian matrix of $V$ at point $P_{0}$ are computed to be

$$
\begin{gathered}
\left.\frac{\partial^{2} V}{\partial \eta^{2}}\right|_{P_{0}}=\frac{\mu^{2}}{s_{\beta} c_{\beta}} \cos \left(\frac{1}{f s_{\beta} c_{\beta}} \hat{h}_{0}\right) \\
\left.\frac{\partial^{2} V}{\partial \eta \partial \hat{h}}\right|_{P_{0}}=0 \\
\left.\frac{\partial^{2} V}{\partial \hat{h}^{2}}\right|_{P_{0}}=\frac{2 \mu^{2}}{s_{\beta} c_{\beta}} \cos \left(\frac{1}{f s_{\beta} c_{\beta}} \hat{h}_{0}\right)-2 \lambda f^{2} \cos \left(\frac{2}{f s_{\beta} c_{\beta}} \hat{h}_{0}\right) \\
+12 \Delta_{0} \hat{h}_{0}^{2}+8 \Delta_{0}^{\prime} \hat{h}_{0}^{3}+\Delta_{0}^{\prime \prime} \hat{h}_{0}^{4}
\end{gathered}
$$

in which

$\Delta_{0} \equiv \Delta\left(\hat{h}_{0}\right),\left.\quad \Delta_{0}^{\prime} \equiv \frac{d \Delta}{d \hat{h}}\right|_{\hat{h}_{0}},\left.\quad \Delta_{0}^{\prime \prime} \equiv \frac{d^{2} \Delta}{d \hat{h}^{2}}\right|_{\hat{h}_{0}}$.

Since the off-diagonal entry of the Hessian matrix is zero, we could read out the pseudoaxion mass $m_{\eta}$ and Higgs mass $m_{h}$ directly from the above equations. The only subtlety is that the $\eta$ field introduced in Eq. (4) is not canonically normalized. It is related to the canonically normalized mass eigenstate field $\eta^{m}$ by a simple rescaling relation [26]

$$
\eta=\eta^{m} \sec \left(\frac{\hat{h}_{0}}{f s_{\beta} c_{\beta}}\right)
$$

With this in mind, the pseudoaxion and Higgs mass squared are found to be

$$
\begin{gathered}
m_{\eta}^{2}=\frac{\mu^{2}}{s_{\beta} c_{\beta}} \sec \left(\frac{\hat{h}_{0}}{f s_{\beta} c_{\beta}}\right) \\
m_{h}^{2}=\frac{\mu^{2}}{s_{\beta} c_{\beta}} \cos \left(\frac{1}{f s_{\beta} c_{\beta}} \hat{h}_{0}\right)-\lambda f^{2} \cos \left(\frac{2}{f s_{\beta} c_{\beta}} \hat{h}_{0}\right) \\
+6 \Delta_{0} \hat{h}_{0}^{2}+4 \Delta_{0}^{\prime} \hat{h}_{0}^{3}+\frac{1}{2} \Delta_{0}^{\prime \prime} \hat{h}_{0}^{4} .
\end{gathered}
$$

We could obtain an $m_{\eta}-m_{h}$ mass relation by eliminating the $\lambda$ in Eq. (51) using the stationary point condition Eq. (43), and the result is (using $v=\sqrt{2} \hat{h}_{0}$ )

$$
\begin{aligned}
m_{\eta}^{2}= & \left\{m_{h}^{2}-v^{2} \Delta_{0}\left[3-\frac{\sqrt{2} v}{f s_{\beta} c_{\beta}} \cot \left(\frac{\sqrt{2} v}{f s_{\beta} c_{\beta}}\right)\right]\right. \\
& \left.-\frac{1}{4} v^{3} \Delta_{0}^{\prime}\left[4 \sqrt{2}-\frac{v}{f s_{\beta} c_{\beta}} \cot \left(\frac{\sqrt{2} v}{f s_{\beta} c_{\beta}}\right)\right]-\frac{1}{8} v^{4} \Delta_{0}^{\prime \prime}\right\} \\
& \times \csc ^{2}\left(\frac{v}{\sqrt{2} f s_{\beta} c_{\beta}}\right) .
\end{aligned}
$$

From Eq. (27) we may easily obtain the following expressions for $\Delta_{0}, \Delta_{0}^{\prime}, \Delta_{0}^{\prime \prime}$ 


$$
\begin{array}{r}
\Delta_{0}=\frac{3}{16 \pi^{2}}\left[\lambda_{t}^{4}\left(\ln \frac{M_{T}^{2}}{m_{t}^{2}}-\frac{1}{2}\right)-\frac{g^{4}}{8}\left(\ln \frac{M_{X}^{2}}{m_{W}^{2}}-\frac{1}{2}\right)\right. \\
\left.-\frac{g^{4}}{16}\left(1+t_{W}^{2}\right)^{2}\left(\ln \frac{M_{Z^{\prime}}^{2}}{m_{Z}^{2}}-\frac{1}{2}\right)\right] \\
\Delta_{0}^{\prime}=-\frac{3 \sqrt{2}}{8 \pi^{2} v}\left[\lambda_{t}^{4}-\frac{g^{4}}{8}-\frac{g^{4}}{16}\left(1+t_{W}^{2}\right)^{2}\right] \\
\Delta_{0}^{\prime \prime}=\frac{3}{4 \pi^{2} v^{2}}\left[\lambda_{t}^{4}-\frac{g^{4}}{8}-\frac{g^{4}}{16}\left(1+t_{W}^{2}\right)^{2}\right] .
\end{array}
$$

The $m_{t}^{2}, m_{W}^{2}, m_{Z}^{2}$ in the expression of $\Delta_{0}$ are field-independent and correspond to the physical mass squared of the top quark, $W$, and $Z$ bosons, respectively. In the mass relation Eq. (52), the field-dependent effects of the field form factor $\Delta(\hat{h})$ are encoded in $\Delta_{0}^{\prime}, \Delta_{0}^{\prime \prime}$.

If we define

$$
\begin{gathered}
\theta \equiv \frac{v}{\sqrt{2} f s_{\beta} c_{\beta}} \\
A \equiv \frac{3}{16 \pi^{2}}\left[\lambda_{t}^{4}-\frac{g^{4}}{8}-\frac{g^{4}}{16}\left(1+t_{W}^{2}\right)^{2}\right] \\
\Delta_{A} \equiv \frac{3}{16 \pi^{2}}\left[\lambda_{t}^{4} \ln \frac{M_{T}^{2}}{m_{t}^{2}}-\frac{g^{4}}{8} \ln \frac{M_{X}^{2}}{m_{W}^{2}}-\frac{g^{4}}{16}\left(1+t_{W}^{2}\right)^{2} \ln \frac{M_{Z^{\prime}}^{2}}{m_{Z}^{2}}\right]
\end{gathered}
$$

then the $m_{\eta}-m_{h}$ mass relation can be written as

$m_{\eta}^{2}=\left[m_{h}^{2}-v^{2} \Delta_{A}\left(3-2 \theta t_{2 \theta}^{-1}\right)+v^{2} A\left(5-2 \theta t_{2 \theta}^{-1}\right)\right] s_{\theta}^{-2}$.

Here $t_{2 \theta}^{-1} \equiv \frac{1}{\tan (2 \theta)}, s_{\theta}^{-2} \equiv \frac{1}{\sin ^{2} \theta}$. Equation (59) is the central result of this paper. If we set $\Delta_{A}=A=0$, then Eq. (59) would yield the corresponding prediction from considering only tree-level scalar potential. The correction to the tree level prediction as exhibited by Eq. (59), is obviously finite and does not depend on the renormalization scale manifestly, consistent with the expectation for a zeroth-order natural relation.

From Eq. (59) we may deduce that $m_{\eta}$ can be predicted once $f, t_{\beta}$, and $m_{T}$ are known. This prediction can be obtained as long as the SLH can be treated as a selfcontained EFT in its domain of validity. It can be tested in a quantitative manner without $a d$ hoc assumptions about UV physics contribution. We see that $m_{\eta}$ and $m_{T}$ are anticorrelated, i.e., with a heavier $m_{T}$ we will obtain a lighter $m_{\eta}$. The electroweak vacuum is supposed to be a local minimum of the scalar effective potential, and therefore we require $m_{\eta}^{2} \geq 0$, which is equivalent to

$$
m_{h}^{2}-v^{2} \Delta_{A}\left(3-2 \theta t_{2 \theta}^{-1}\right)+v^{2} A\left(5-2 \theta t_{2 \theta}^{-1}\right) \geq 0 .
$$

This condition sets an upper bound on $m_{T}$ when $f, t_{\beta}$ are given. The parameter $M_{T}$ also has a lower bound for fixed $f, t_{\beta}[34]$

$$
M_{T} \geq \sqrt{2} \frac{m_{t}}{v} f s_{2 \beta} \approx f s_{2 \beta}
$$

where $s_{2 \beta} \equiv \sin (2 \beta)$. This bound comes from the definition of $M_{T}$ in Eq. (32) and the requirement of the top Yukawa $\lambda_{t}$ in Eq. (28) to yield the correct top quark mass.

\section{Unitarity constraint}

Due to the nonlinearly realized scalar sector, the SLH can at best be seen as an EFT valid up to some cutoff scale. Apart from NDA consideration which yields a cutoff of $4 \pi f c_{\beta}$, we may also consider the bound from perturbative unitarity, which is usually expected to yield a more stringent constraint compared to NDA [23].

To derive the perturbative unitarity constraint for the SLH, we adopt the methodology of Ref. [23]. To simplify the problem, we only consider the kinetic terms of the nonlinear sigma fields, in the limit of vanishing EWSB. In the SLH we have two copies of $S U(3) \rightarrow S U(2)$ global symmetry breaking, realized by $\Phi_{1}$ and $\Phi_{2}$ respectively, and the only relevant difference between them is the symmetry breaking scale, $f c_{\beta}$ for $\Phi_{1}$ and $f s_{\beta}$ for $\Phi_{2}$. In our setting $\Phi_{1}$ is chosen to be the one with a lower symmetry breaking scale (i.e., $t_{\beta} \geq 1$ ), and therefore we only consider the perturbative unitarity constraint from analysis of kinetic terms of $\Phi_{1}$.

For the perturbative unitarity analysis, it is convenient to parametrize $\Phi_{1}$ as $\left(f_{1} \equiv f c_{\beta}\right)$

$$
\Phi_{1}=\exp \left(\frac{i \Theta_{1}}{f_{1}}\right)\left(\begin{array}{c}
0 \\
0 \\
f_{1}
\end{array}\right)
$$

in which

$$
\Theta_{1}=\frac{1}{\sqrt{2}}\left(\begin{array}{ccc}
\frac{1}{2} \pi_{8} & 0 & \pi_{4}-i \pi_{5} \\
0 & \frac{1}{2} \pi_{8} & \pi_{6}-i \pi_{7} \\
\pi_{4}+i \pi_{5} & \pi_{6}+i \pi_{7} & -\pi_{8}
\end{array}\right)
$$

The kinetic Lagrangian of $\Phi_{1}$ can now be written as 


$$
\begin{aligned}
\mathcal{L}_{k 1}= & \left(\partial_{\mu} \Phi_{1}\right)^{\dagger} \partial^{\mu} \Phi_{1} \\
= & \frac{1}{2} \sum_{a} \partial_{\mu} \pi_{a} \partial^{\mu} \pi_{a}+\frac{1}{4 \sqrt{2} f_{1}}\left(\pi_{4} \partial_{\mu} \pi_{5}-\pi_{5} \partial_{\mu} \pi_{4}+\pi_{6} \partial_{\mu} \pi_{7}-\pi_{7} \partial_{\mu} \pi_{6}\right) \partial^{\mu} \pi_{8} \\
& +\sum_{i<j}\left\{-\frac{1}{12 f_{1}^{2}}\left[\pi_{i}^{2}\left(\partial_{\mu} \pi_{j}\right)^{2}+\pi_{j}^{2}\left(\partial_{\mu} \pi_{i}\right)^{2}\right]+\frac{1}{6 f_{1}^{2}}\left(\pi_{i} \partial_{\mu} \pi_{i}\right)\left(\pi_{j} \partial^{\mu} \pi_{j}\right)\right\} \\
& +\sum_{i}\left\{-\frac{3}{64 f_{1}^{2}} \pi_{8}^{2}\left(\partial_{\mu} \pi_{i}\right)^{2}-\frac{7}{64 f_{1}^{2}}\left(\partial_{\mu} \pi_{8}\right)^{2} \pi_{i}^{2}+\frac{5}{32 f_{1}^{2}}\left(\pi_{8} \partial_{\mu} \pi_{8}\right)\left(\pi_{i} \partial^{\mu} \pi_{i}\right)\right\} .
\end{aligned}
$$

In the above equation, the ranges of summation are $a=4$, 5, 6, 7, 8 and $i, j=4,5,6,7$. Considering $\pi_{a} \pi_{a} \rightarrow \pi_{b} \pi_{b}$ scattering (with $a, b=4,5,6,7,8$ ), the 0th partial wave amplitude matrix is then computed to be

$$
\mathcal{A}_{0}=\frac{s}{64 \pi f_{1}^{2}}\left(\begin{array}{ccccc}
0 & 1 & 1 & 1 & 1 \\
1 & 0 & 1 & 1 & 1 \\
1 & 1 & 0 & 1 & 1 \\
1 & 1 & 1 & 0 & 1 \\
1 & 1 & 1 & 1 & 0
\end{array}\right) \text {. }
$$

The eigenvalues of $\mathcal{A}_{0}$ are

$$
a_{0 j}=\frac{s}{64 \pi f_{1}^{2}}(4,-1,-1,-1,-1) .
$$

Requiring $\left|\operatorname{Re}\left(a_{0 j}\right)\right| \leq \frac{1}{2}$, we are able to obtain the perturbative unitarity constraint

$$
\sqrt{s} \leq \sqrt{8 \pi} f_{1}
$$

As expected, this turns out to be more stringent than the NDA bound of $\sqrt{s} \leq 4 \pi f_{1}$. In the following analysis, we require the particle masses that are relevant to our scalar potential analysis be smaller than the unitarity cutoff $\sqrt{8 \pi} f_{1}$. Specifically, we require (recall $f_{1} \equiv f c_{\beta}$ )

$$
\begin{aligned}
& M_{Z^{\prime}} \leq \sqrt{8 \pi} f c_{\beta} \\
& M_{T} \leq \sqrt{8 \pi} f c_{\beta} .
\end{aligned}
$$

In the SLH, $Z^{\prime}$ is heavier than $X, Y$ bosons, therefore we impose the constraint on $M_{Z^{\prime}}$. The difference between $M_{Z^{\prime}}$ and $m_{Z^{\prime}}$ and the difference between $M_{T}$ and $m_{T}$ are both small, and in the unitarity constraint we use $M_{Z^{\prime}}$ and $M_{T}$ for simplicity. We can use Eq. (31) to find a constraint on $t_{\beta}$ from Eq. (68). This constraint when combined with the assumption of $t_{\beta} \geq 1$, implies the following allowed region of $t_{\beta}$ which we assume hereafter

$$
1 \leq t_{\beta} \leq \sqrt{\frac{4 \pi\left(3-t_{W}^{2}\right)}{g^{2}}-1}
$$

The unitarity constraint yields an upper bound on $t_{\beta}$, which is not difficult to understand. $t_{\beta}$ can be viewed as a measure of the asymmetry between the vacuum expectation values of $\Phi_{1}$ and $\Phi_{2}$. The unitarity constraint depends on the smaller of the two vacuum expectation values, while the $Z^{\prime}$ boson mass depends on their quadrature. Therefore the asymmetry between the two vacuum expectation values cannot be too large.

\section{Allowed region of parameter space}

With the results from the scalar potential analysis and unitarity constraints obtained in this section, we are ready to make plots characterizing the allowed region of parameter space with an understanding of its basic features. However, before that we note there are some technicalities related to input parameter corrections. More specifically, the parameters $g, v, t_{W}, \lambda_{t}$ that appear in our parametrization of the SLH should not be directly identified with the corresponding quantities in the SM, which we denote as $g_{\mathrm{SM}}, v_{\mathrm{SM}}, t_{W, \mathrm{SM}}, \lambda_{t, \mathrm{SM}}$, respectively. Their relations should be established by producing a common set of wellmeasured physical observables. In this paper, we are concerned with correction due to the SLH, rather than higher order radiative corrections. Thus we opt to work at tree level but retain the leading $\mathcal{O}\left(\frac{v^{2}}{f^{2}}\right)$ corrections. The correspondence turns out to be

$$
\begin{gathered}
g=g_{\mathrm{SM}}\left(1+\frac{1}{4 t_{\beta}^{2}} \frac{v_{\mathrm{SM}}^{2}}{f^{2}}\right) \\
v=v_{\mathrm{SM}}\left(1+\frac{t_{\beta}^{4}-t_{\beta}^{2}-2}{12 t_{\beta}^{2}} \frac{v_{\mathrm{SM}}^{2}}{f^{2}}\right) \\
t_{W}=t_{W, \mathrm{SM}}\left(1-\frac{1+t_{W, \mathrm{SM}}^{2}}{4 t_{\beta}^{2}} \frac{v_{\mathrm{SM}}^{2}}{f^{2}}\right) \\
\lambda_{t}=\lambda_{t, \mathrm{SM}}\left[1+\left(\frac{1}{4 s_{\beta}^{2}}-\frac{\lambda_{t, \mathrm{SM}}^{2}}{4} \frac{f^{2}}{M_{T}^{2}}\right) \frac{v_{\mathrm{SM}}^{2}}{f^{2}}\right]
\end{gathered}
$$



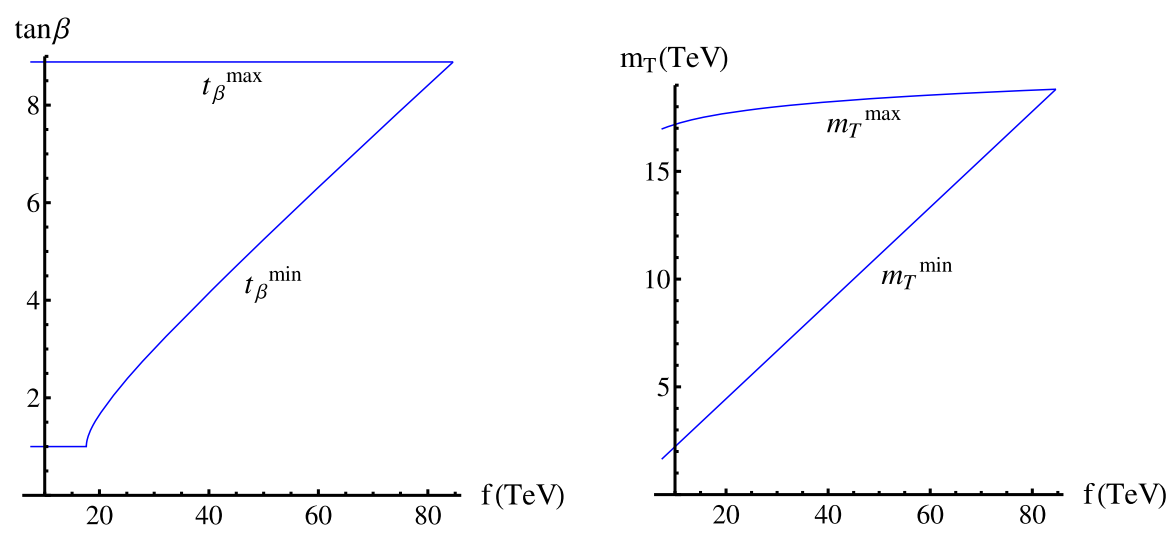

FIG. 1. Left: Maximum and minimum $t_{\beta}$ value as a function of $f$. Right: Maximum and minimum $m_{T}$ value as a function of $f$.

in which we use the following values for SM quantities

$$
\begin{array}{rlrl}
g_{\mathrm{SM}} & =0.653, & & v_{\mathrm{SM}}=246.2 \mathrm{GeV} \\
t_{W, \mathrm{SM}} & =0.536, & \lambda_{t, \mathrm{SM}}=0.995
\end{array}
$$

The $Z^{\prime}$ boson in the SLH is subject to the stringent constraint from the LHC search of a high-mass resonance decaying into dilepton. Using ATLAS results [35] we set a crude lower bound on $f$ as [27]

$$
f>7.5 \mathrm{TeV} \text {. }
$$

With such a stringent constraint the effect of $\mathcal{O}\left(\frac{v^{2}}{f^{2}}\right)$ corrections is in fact very small. Nevertheless we take them into account in our numerical analysis to validate the stability of our results against these corrections.

In Fig. 1 we plot the maximum and minimum allowed $t_{\beta}$ and $m_{T}$ values as a function of $f$. Constraints from Eqs. (60), (61), (70), and (69) are taken into account. From the left panel of Fig. 1 we see a constant $t_{\beta}^{\max }$ value $\left(t_{\beta}^{\max } \approx 9\right)$ for all $f$. This follows from Eq. (70) which requires $Z^{\prime}$ mass should not exceed the unitarity constraint.

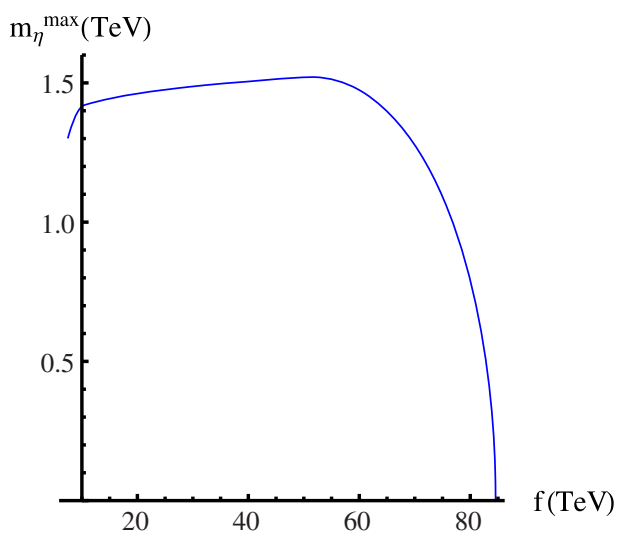

FIG. 2. Maximum $m_{\eta}$ value as a function of $f$.
Since $t_{\beta}$ is bounded from above, Eq. (61) then imposes a lower bound on top partner mass for fixed $f$. This explains the $m_{T}^{\min }$ curve shown in the right panel of Fig. 1. The $m_{T}^{\max }$ curve in the right panel of Fig. 1 is determined by Eq. (60), reflecting the fact that a too heavy top partner could lead to a negative pseudoaxion mass squared. The upper bound on heavy top partner mass should be larger than its lower bound, which leads to the increasing of $t_{\beta}^{\min }$ for larger $f$ as shown in the left panel of Fig. 1. Therefore, with the increase of $f$, the allowed range of variation for $t_{\beta}$ shrinks, and eventually hit a point at $f \approx 85 \mathrm{TeV}$, above which a perturbative treatment of the SLH as an EFT might not be reliable. We remark here that it is important to retain the field dependence in the field form factor $\Delta(\hat{h})$ in order to obtain reliable estimates of $m_{T}^{\max }$ and $m_{\eta}-m_{T}$ mass relation. Although the difference in treating $\Delta(\hat{h})$ as field-independent and field-dependent is proportional to a small quantity $A \approx 0.018$, the $M_{T}$ parameter enters as the

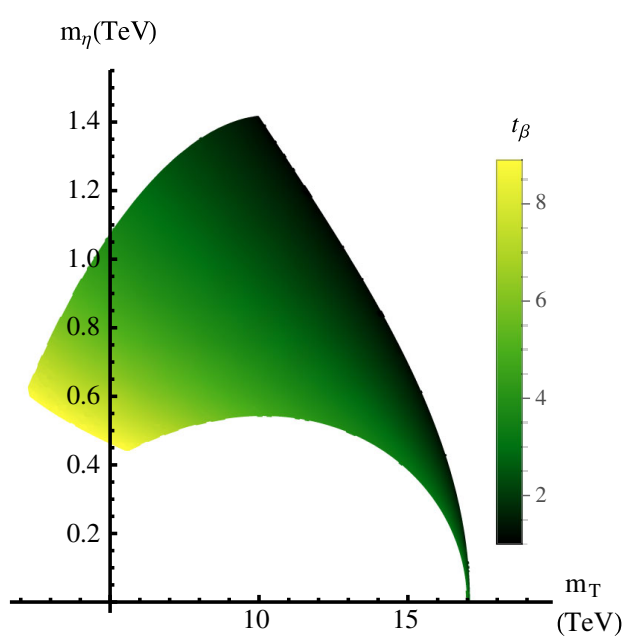

FIG. 3. $t_{\beta}$ density plot in the allowed region in the $m_{\eta}-m_{T}$ plane for $f=10 \mathrm{TeV}$. 

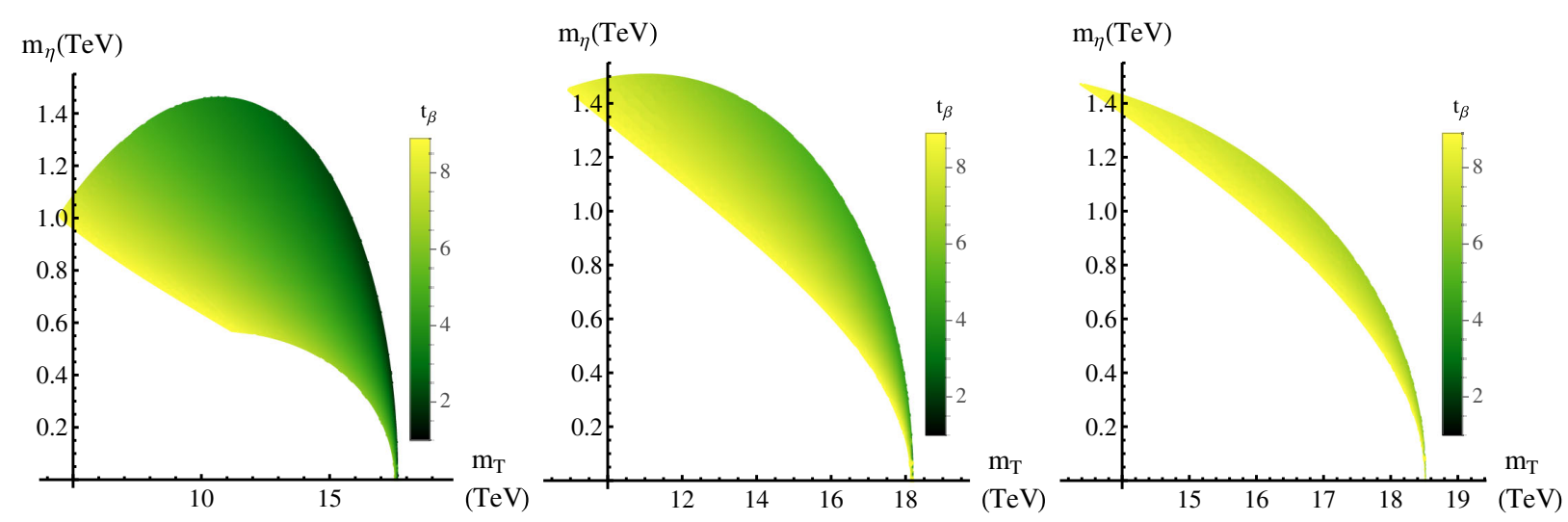

FIG. 4. $t_{\beta}$ density plot in the allowed region in the $m_{\eta}-m_{T}$ plane for $f=20,40,60 \mathrm{TeV}$.

argument of a logarithmic function and is therefore very sensitive to such corrections. If we treated $\Delta(\hat{h})$ as field-independent, we would have obtained a maximum $m_{T}^{\max }$ value of about half of the value obtained by retaining field-dependence.

In Fig. 2 we plot the maximum allowed $m_{\eta}$ value as a function of $f$. The minimum allowed $m_{\eta}$ value, before any experimental or observational constraint is taken into account, is zero. The maximum allowed $m_{\eta}$ for fixed $f$ reaches a maximum at about $f \approx 51 \mathrm{TeV}$, $m_{\eta}=1.5 \mathrm{TeV}$.

Figure 3 exhibits the $t_{\beta}$ value in the allowed region in the $m_{\eta}-m_{T}$ plane for $f=10 \mathrm{TeV}$. One easy way to understand this figure is to follow the contour of constant $t_{\beta}$, which can be identified by the color code. If we start from a high value of $t_{\beta}$ at some point with lighter color, then we can move along two opposite directions by keeping $t_{\beta}$ fixed. We may move along the direction which increases $m_{T}$ and eventually reach a point that saturates the unitarity bound Eq. (69), which is responsible for the dent in the lower part of the figure. On the other hand, we may move along the direction which decreases $m_{T}$ and eventually reach a point that saturates the bound in Eq. (61), which determines the northwest boundary of the allowed region. For $f=10 \mathrm{TeV}$, the northeast and southwest boundary of the allowed region are cut off by $t_{\beta}=1$ and $t_{\beta}=t_{\beta}^{\max } \approx 9$, respectively.

In Fig. 4 we present the $t_{\beta}$ density plot in the allowed region in the $m_{\eta}-m_{T}$ plane for $f=20$, $40,60 \mathrm{TeV}$. Because of the increased $t_{\beta}^{\min }$ value, the boundary of the upper half allowed region will always be determined by Eq. (61) and it will not be cut off by $t_{\beta}=1$. This explains the absence of a sharp turning point in the upper boundary of the allowed region in Fig. 4. Moreover, the effect of unitarity constraint on $m_{T}$ becomes milder, and vanishes for $f=40,60 \mathrm{TeV}$ cases, explaining the shrinking and disappearance of the dent in the lower part.

\section{NATURALNESS IN THE SIMPLEST LITTLE HIGGS}

\section{A. Anatomy of naturalness in continuum effective field theory}

We are now prepared to analyze the fine-tuning problem in a general manner within the CEFT framework. ${ }^{6} \mathrm{~A}$ global physical picture suggested by CEFT is that between thresholds, the renormalization structure of a QFT is encoded in its RGE in a mass-independent renormalization scheme, while when going below a mass threshold, heavy degrees of freedom should be integrated out and the switch to a low energy effective QFT should be made in order to facilitate an easy grasp of the main feature of the theory. Accordingly, two sources of physical fine-tuning in this global picture can be identified. First, there can be finetuning associated with the RG running between thresholds (referred to as "RG tuning"). This happens if the value of a running parameter is very small at a particular renormalization scale for observation while its running is very fast (i.e., $\beta$ function is large). In such a case when we consider a finite range of RG flow then it is obvious that there can be a high sensitivity of the IR parameter to the UV parameter. A simple example is scalar QED, in which if a scalar quartic interaction is not written down, it can still be generated via RG running, and therefore requiring the quartic to vanish at a particular point would be a type of fine-tuning. We note that such kind of fine-tuning in the context of Little Higgs models has been pointed out before [37]. Second, there can be fine-tuning associated with the transition from the EFT above the threshold to the EFT below the threshold (referred to as "threshold tuning"). Generally speaking, the degrees of freedom and the

\footnotetext{
${ }^{6}$ In this work, we quantify the degree of fine-tuning as the sensitivity of IR physical parameters to UV physical parameters. Sensitivity to unphysical parameters such as the regularization cutoff is not considered as fine-tuning and is thus excluded from discussion. This is in accordance with the discussion in, e.g., Ref. [36].
} 
description of the theory change due to crossing the threshold. This change of description could induce additional sensitivity of IR parameters to UV parameters. We will see at below how the threshold tuning in the SLH can be identified.

\section{B. Fine-tuning in the SLH}

The SLH can be considered an EFT valid up to its unitarity cutoff $\Lambda_{U} \equiv \sqrt{8 \pi} f c_{\beta}$. Roughly speaking, there is a scale separation in the validity range of the SLH. Two characteristic scales can be identified, one is associated with the scales of heavy sector particles, such as $m_{T}, m_{Z^{\prime}}$, the other is the electroweak scale which can be represented by $v$ or $m_{h}$. The fine-tuning in the SLH, from the CEFT point of view, is about how the electroweak scale parameters, such as $v$ or $m_{h}$, are sensitive to high scale parameters in the model. Here the "high scale" is naturally chosen to be the highest scale at which the SLH claims to be valid as an EFT. In our analysis this is unsurprisingly chosen to be the unitarity cutoff $\Lambda_{U}$. For simplicity we ignore small input parameter corrections in the fine-tuning analysis.

The values of $v$ and $m_{h}$ can be calculated once the following set of parameters are given at $\Lambda_{U}: f, t_{\beta}$, $M_{T}, \lambda_{R}, \mu^{2}$. In our analysis we neglect field strength renormalization effects and the only running parameter in this set is $\lambda_{R}$. The procedure to calculate $v$ and $m_{h}$ is then straightforward: we first follow the RG flow to a scale $M_{L}$ which satisfies $\bar{\lambda}=0$ at $\mu_{R}=M_{L}$ [ $\bar{\lambda}$ is defined by Eq. (22)]. At $\mu_{R}=M_{L}$ we have the parameter $\lambda$ introduced in Eq. (24) just given by $\lambda_{R}$. Next we may use the analysis in the previous section to obtain $v$ and $m_{h}$, which can be viewed as transition to a low energy EFT for the electroweak scale. The RG tuning and threshold tuning can be obtained respectively from the above two steps.

Let us first investigate the threshold tuning. This requires us to express $v$ and $m_{h}$ in terms of $\lambda, \mu^{2}$ and $f, t_{\beta}, M_{T}$. From the stationary point condition Eq. (43) we could obtain the following expression of $v^{2}$ by expanding the sines to $\hat{h}_{0}^{3}$

$$
v^{2}=\frac{4\left(\lambda f^{2}-\frac{\mu^{2}}{s_{\beta} c_{\beta}}\right)}{\frac{1}{3 f^{2} s_{\beta}^{2} c_{\beta}^{2}}\left(4 \lambda f^{2}-\frac{\mu^{2}}{s_{\beta} c_{\beta}}\right)+4 \Delta_{0}-2 A}
$$

in which $\Delta_{0}$ and $A$ are given by Eqs. (53) and (57), respectively. Then we may expand the cosines in Eq. (51) to $\hat{h}_{0}^{2}$ and plug in Eq. (77) to obtain

$$
m_{h}^{2}=2\left(\lambda f^{2}-\frac{\mu^{2}}{s_{\beta} c_{\beta}}\right)-2 A v^{2}
$$

in which $v^{2}$ is given by Eq. (77). Let us define threshold tuning $\Delta_{\mathrm{TH}}^{\lambda}, \Delta_{\mathrm{TH}}^{\mu^{2}}$ for $\lambda$ and $\mu^{2}$ as follows (with inspiration from Ref. [38])

$$
\begin{gathered}
\Delta_{\mathrm{TH}}^{\lambda} \equiv\left|\frac{\lambda}{m_{h}^{2}} \frac{\partial m_{h}^{2}}{\partial \lambda}\right| \\
\Delta_{\mathrm{TH}}^{\mu^{2}} \equiv\left|\frac{\mu^{2}}{m_{h}^{2}} \frac{\partial m_{h}^{2}}{\partial \mu^{2}}\right| .
\end{gathered}
$$

These definitions obviously reflect how the relative variation of $m_{h}^{2}$ is sensitive to the relative variation of $\lambda$ and $\mu^{2}$. From Eq. (78) the threshold tuning values are calculated to be

$$
\begin{gathered}
\Delta_{\mathrm{TH}}^{\lambda}=1+\frac{2 m_{\eta}^{2}}{m_{h}^{2}} \\
\Delta_{\mathrm{TH}}^{\mu^{2}}=\frac{2 m_{\eta}^{2}}{m_{h}^{2}} .
\end{gathered}
$$

In obtaining the above threshold tuning values we have neglected terms proportional to $A$ or relatively suppressed by $\frac{v^{2}}{f^{2}}$. Also, we checked that if we use $v^{2}$ instead of $m_{h}^{2}$ to quantify the threshold tuning, the results do not change. The above results suggest that the threshold tuning is determined by $m_{\eta}$ : with larger $m_{\eta}$ we get larger tuning. This is easy to understand since from Eq. (78) we see that $m_{h}^{2}$ can be approximately viewed as the result of cancellation between $2 \lambda f^{2}$ and $\frac{2 \mu^{2}}{s_{\beta} c_{\beta}} \sim 2 m_{\eta}^{2}$.

To calculate the RG tuning we need the $\beta$ function of $\lambda_{R}$, denoted as $\beta_{\lambda}$. In this work we neglect the contribution to $\beta_{\lambda}$ from field strength renormalization and scalar loop. From Eq. (26) the following expression for $\beta_{\lambda}$ can be derived

$$
\beta_{\lambda}=-\frac{3 \lambda_{t}^{2}}{4 \pi^{2}} \frac{M_{T}^{2}}{f^{2}}+\frac{3 g^{4}}{32 \pi^{2}} \frac{5+t_{W}^{2}}{3-t_{W}^{2}} .
$$

We use $\lambda_{U}$ to denote the value of $\lambda_{R}$ defined at the unitarity cutoff $\Lambda_{U}$. Then the relation between $\lambda$ and $\lambda_{U}$ can be expressed as

$$
\lambda=\lambda_{U}-\beta_{\lambda} \ln \frac{\Lambda_{U}}{M_{L}} .
$$

The RG tuning of $\lambda$ is then defined through

$$
\Delta_{\mathrm{RG}}^{\lambda} \equiv\left|\frac{\lambda_{U}}{\lambda} \frac{\partial \lambda}{\partial \lambda_{U}}\right|=\left|1+\frac{1}{\lambda} \beta_{\lambda} \ln \frac{\Lambda_{U}}{M_{L}}\right| .
$$

The special scale $M_{L}$ is defined by making $\bar{\lambda}$ vanish. From this requirement we find the following useful relation for analysis of the RG tuning

$$
\left[\lambda_{t}^{2} M_{T}^{2}-\frac{5+t_{W}^{2}}{8\left(3-t_{W}^{2}\right)} g^{4} f^{2}\right] \ln \frac{M_{T}}{M_{L}}=\frac{1}{2} B
$$




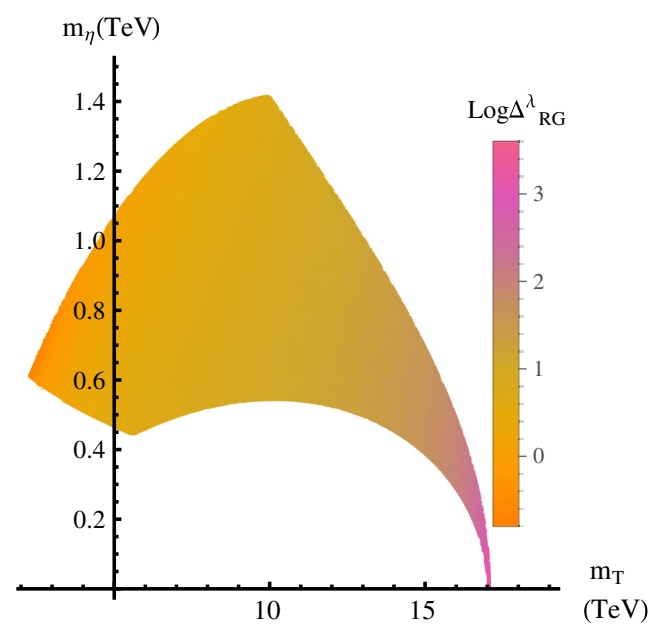

FIG. 5. Density plot of $\log \Delta_{\mathrm{RG}}^{\lambda}$ in the $m_{\eta}-m_{T}$ plane for $f=10 \mathrm{TeV}$. Here Log means $\log _{10}$.

where $B$ is defined as

$$
\begin{aligned}
B \equiv & \lambda_{t}^{2} M_{T}^{2}+\frac{1}{4} g^{2} M_{X}^{2}\left(\ln \frac{M_{X}^{2}}{M_{T}^{2}}-\frac{1}{3}\right) \\
& +\frac{1}{8} g^{2}\left(1+t_{W}^{2}\right) M_{Z^{\prime}}^{2}\left(\ln \frac{M_{Z^{\prime}}^{2}}{M_{T}^{2}}-\frac{1}{3}\right) .
\end{aligned}
$$

$\Delta_{\mathrm{RG}}^{\lambda}$ is then calculated to be $\left(\Lambda_{U}=\sqrt{8 \pi} f c_{\beta}\right)$

$\Delta_{\mathrm{RG}}^{\lambda}=\left|1-\frac{3}{2 \pi^{2}} \frac{\lambda_{t}^{2} M_{T}^{2}-\frac{g^{4} f^{2}}{8} \frac{5+t_{W}^{2}}{3-t_{W}^{2}}}{m_{h}^{2}+2 m_{\eta}^{2}} \ln \frac{\sqrt{8 \pi} f c_{\beta}}{M_{T}}-\frac{3}{4 \pi^{2}} \frac{B}{m_{h}^{2}+2 m_{\eta}^{2}}\right|$.

To obtain this expression we have made use of Eq. (78) to find the $\lambda$ expressed by $m_{h}$ and $m_{\eta}$ and again neglected corrections suppressed by $\frac{v^{2}}{f^{2}}$ or $A$.

Figure 5 presents the density plot of $\log \Delta_{\mathrm{RG}}^{\lambda}$ in the $m_{\eta}-m_{T}$ plane for $f=10 \mathrm{TeV}$. We note that in the left region where $m_{T}$ is light, $\Delta_{\mathrm{RG}}^{\lambda}$ can drop below 1 , but not reach 0 . We have checked that for $f=10 \mathrm{TeV}, \lambda_{U}$ is always negative. Figure 5 can be regarded alternatively as an indication of how negative $\lambda_{U}$ is. For large $\Delta_{\mathrm{RG}}^{\lambda}$ it is therefore natural to question the vacuum stability of the corresponding parameter point. Because there still exists parameter region of relatively small $\Delta_{\mathrm{RG}}^{\lambda}$ we do not expect in any case that vacuum stability consideration should exclude all the allowed parameter space in Fig. 5, although a detailed study is beyond the scope of the present paper.

The RG tuning of $\mu^{2}$ parameter denoted as $\Delta_{\mathrm{RG}}^{\mu^{2}}$ can also be discussed, but this turns out to be trivial, i.e.,

$$
\Delta_{\mathrm{RG}}^{\mu^{2}}=1
$$

since the $\mu_{R}^{2}$ does not run (when wave-function renormalization and small contribution from light Yukawa are neglected).

The threshold tuning and RG tuning derived above can be combined to define a total tuning of a given parameter (e.g., $\lambda$ or $\mu^{2}$ ). For example, the total tuning of $\lambda$ is defined as

$$
\Delta_{\mathrm{TOT}}^{\lambda} \equiv \Delta_{\mathrm{TH}}^{\lambda} \times \Delta_{\mathrm{RG}}^{\lambda}
$$

The use of multiplication in the above definition is easy to understand: the total tuning defined in this way just reflects how the relative change of $m_{h}^{2}$ is sensitive to the relative change of $\lambda_{U}$. For $\mu^{2}$, we have

$$
\Delta_{\mathrm{TOT}}^{\mu^{2}} \equiv \Delta_{\mathrm{TH}}^{\mu^{2}} \times \Delta_{\mathrm{RG}}^{\mu^{2}}=\Delta_{\mathrm{TH}}^{\mu^{2}}
$$

where the second step is due to Eq. (89). Finally, to quantify the overall degree of fine-tuning in the $\mathrm{SLH}$, we define

$$
\Delta_{\mathrm{TOT}}=\max \left\{\Delta_{\mathrm{TOT}}^{\mu^{2}}, \Delta_{\mathrm{TOT}}^{\lambda}\right\} .
$$

For simplicity we do not attempt a more sophisticated statistical combination.

Let us take a closer look at $\Delta_{\mathrm{TOT}}^{\lambda}$, which is easily calculated to be

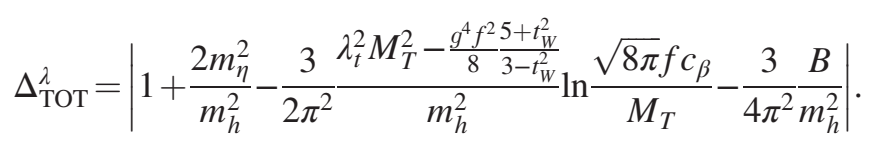

It is worth noticing that part of the above equation (the term containing $\ln \frac{\sqrt{8 \pi} f c_{\beta}}{M_{T}}$ ) is very similar to the fine-tuning definition employed in Ref. [15], which we copy here for convenience $^{7}$ (see Eqs. (4.2) and (4.3) in Ref. [15])

$\Delta=\frac{\left|\delta \mu^{2}\right|}{\mu_{\mathrm{obs}}^{2}}, \quad \mu_{\mathrm{obs}}^{2}=\frac{m_{h}^{2}}{2}, \quad \delta \mu^{2}=-\frac{3 \lambda_{t}^{2} m_{T}^{2}}{8 \pi^{2}} \log \frac{\Lambda^{2}}{m_{T}^{2}}$

In the above equation the notations are in accord with Ref. [15]. Furthermore, Ref. [15] uses $\Lambda=4 \pi f$ to cut off the divergent one-loop integral. We see that if in our expression of $\Delta_{\text {TOT }}^{\lambda}$, we only retain the term containing $\ln \frac{\sqrt{8 \pi} f c_{\beta}}{M_{T}}$, neglect the $\frac{g^{4} f^{2}}{8} \frac{5+t_{W}^{2}}{3-t_{W}^{2}}$ part, and let $\Lambda_{U}=\sqrt{8 \pi} f c_{\beta} \rightarrow \Lambda=4 \pi f$, then we recover the fine-tuning definition of Ref. [15]. The differences between our treatment and the definition in Ref. [15] deserves some comments. The use of unitarity cutoff $\Lambda_{U}$ instead of NDA cutoff $\Lambda$ is not essential, although this could have some

\footnotetext{
${ }^{7}$ A similar definition is also employed by Ref. [39] in the context of the littlest Higgs model with T-parity.
} 
impact on the quantitative value of fine-tuning. The $\frac{g^{4} f^{2}}{8} \frac{5+t_{W}^{2}}{3-t_{W}^{2}}$ part reflects the interpretational difference between our treatment and that of Ref. [15]. In Ref. [15], the regularization cutoff is invested with a physical meaning and the different terms in the one-loop $\delta \mu^{2}$ expression are then considered as independent sources of tuning. However, in our treatment, this expression reflects the RG running of $\lambda_{R}$ and through this running we infer how the IR parameters are sensitive to the UV parameters. It is then clear that the $\frac{g^{4} f^{2}}{8} \frac{5+t_{W}^{2}}{3-t_{W}^{2}}$ part should be retained because it also contributes to the $\beta$ function. Since this part has a relative minus sign compared to the fermionic contribution, it effectively reduces the fine-tuning in the model. In Eq. (93) we also have a $\frac{3}{4 \pi^{2}} \frac{B}{m_{h}^{2}}$ term which is absent in the definition of Ref. [15]. This is again due to the interpretational difference. In our treatment, the starting point of the RG running is $M_{L}$, and only when the fermionic contribution is much larger than the gauge contribution to the scalar effective potential can we have $M_{L} \sim M_{T}$. Finally, in Eq. (93) we have the $1+\frac{2 m_{n}^{2}}{m_{h}^{2}}$ part which is also absent in Ref. [15]. We can understand the role of this part in the following manner. Suppose we turn off the $1+\frac{2 m_{n}^{2}}{m_{h}^{2}}$ part for the moment, then we could realize that the situation would correspond to when defining the RG tuning $\Delta_{\mathrm{RG}}^{\lambda}$ we omit the "1" before the plus sign in Eq. (85). In other words, the ratio of the amount of RG running to the value of $\lambda_{R}$ defined at $M_{L}$ is taken to be the measure of $R G$ tuning. If $R G$ tuning is much larger than 1 , then the difference induced by the $1+\frac{2 m_{n}^{2}}{m_{h}^{2}}$ part is in fact negligible.

The above discussion in fact leads to a more clear understanding of the NDCC assumption. In previous literature, this is equivalent to calculating the scalar effective potential by turning off the relevant tree-level contribution and imposing a NDA cutoff to momentum integral. In our fine-tuning analysis we see that for those parameter points that satisfy $\lambda_{U}=0$, the total tuning of $\lambda$, denoted as $\Delta_{\text {TOT }}^{\lambda}$ vanishes since the RG tuning $\Delta_{\mathrm{RG}}^{\lambda}$ vanishes. Therefore, if we interpret the NDCC assumption as corresponding to $\lambda_{U}=0$ in the CEFT approach, then parameter points that satisfy this assumption will automatically have the property of making $\Delta_{\text {TOT }}^{\lambda}=0$. It is tempting to consider these parameter points satisfying the NDCC assumption as particularly good ones. However, the real situation is not that simple, due to the following reasons. First, even if $\Delta_{\text {TOT }}^{\lambda}=0$, we still need to consider $\Delta_{\text {TOT }}^{\mu^{2}}$, which cannot be made arbitrarily small given $\Delta_{\text {TOT }}^{\lambda}=0$. Second, $\Delta_{\text {TOT }}^{\lambda}=0$ is, honestly speaking, illusory. This is because $\Delta_{\text {TOT }}^{\lambda}=0$ is derived from a completely IR point of view. If we think a little bit about UV completion, then from the UV point of view, any tiny variation of UV fixed that is possible to vary $\lambda_{U}$ slightly indicates an infinite sensitivity and thus an infinite amount of fine-tuning. The problem is that we choose a "bad" transition point to connect IR and UV. If we use another scale at which $\lambda_{U} \neq 0$, we would be able to obtain finite fine-tuning result.

It is also instructive at the moment to consider the alternative interpretation [20] that $\Lambda$ is chosen to be the renormalization scale in conjunction with the NDCC assumption adopted in previous literature. In our CEFT approach the renormalization scale is denoted as $\mu_{R}$ [Eq. (26)] and physics does not depend on the choice of $\mu_{R}$. In fact we obtained the $\beta$ function associated with $\lambda$ and the renormalized coupling $\lambda_{R}$ is required to have the correct $\mu_{R}$ dependence to cancel the $\mu_{R}$ dependence in $\bar{\lambda}$. Then for fixed chosen cutoff, the solution under the NDCC assumption obtained by previous literature would correspond to a subset of the solution obtained in our CEFT approach. The description of this subset of solution, from the CEFT point of view, is a little bit subtle since it seems to violate closure under renormalization. However, if we pick any one element of this subset, it does not violate RG invariance: at the cutoff $\lambda_{R}$ vanishes and only receives loop corrections, while at scale below cutoff $\lambda_{R}$ receives both tree and loop contribution. The CEFT approach makes this point clear. However, we note that this alternative interpretation can only hold when the replacement $\Lambda \rightarrow \mu_{R}$ indeed yields the corresponding CEFT result. In those circumstances which terms involving $\Lambda^{2}$ are retained, it is difficult to reconcile this interpretation with a massindependent renormalization scheme.

Therefore it is clarified that the commonly seen NDCC assumption actually corresponds to selecting a subset of parameter space obtained via the CEFT approach, which satisfies $\lambda_{U}=0$. We need to be careful about such selection, for the following reasons. First, we need to check whether such selection is consistent with the requirement of correct EWSB, given the measured electroweak vacuum expectation value and Higgs mass. Second, there is no a priori reason to confine us to this selection. The nature may well allow some amount of $\Delta_{\text {TOT }}^{\lambda}$. Third, the real EFT cutoff is unknown, and the physical predictions made based on some fixed $\Lambda$ value could be unreliable. This is the crucial place where CEFT reveals its power: as long as we accept the SLH as a self-contained low energy EFT where perturbation theory is valid, we are able to establish the mass relation like Eq. (59) without reliance on the knowledge or assumption about the cutoff.

In Fig. 6 we present the density plot of $\log \Delta_{\text {TOT }}^{\lambda}$ and $\log \Delta_{\mathrm{TOT}}$ in the $m_{\eta}-m_{T}$ plane for $f=10 \mathrm{TeV}$. Two plots are similar except for a small region in the leftmost corner. This is because for $f=10 \mathrm{TeV} \Delta_{\text {TOT }}$ is mainly determined by $\Delta_{\text {TOT }}^{\lambda}$ except for the region with small enough $m_{T}$ in which $\Delta_{\text {TOT }}^{\lambda}<\Delta_{\text {TOT }}^{\mu^{2}}$. From the figure we see that for $f=$ $10 \mathrm{TeV}$ the parameter space favored by naturalness consideration has small $m_{T}$ (down to $\sim 3 \mathrm{TeV}$ ) and large $t_{\beta}$ 

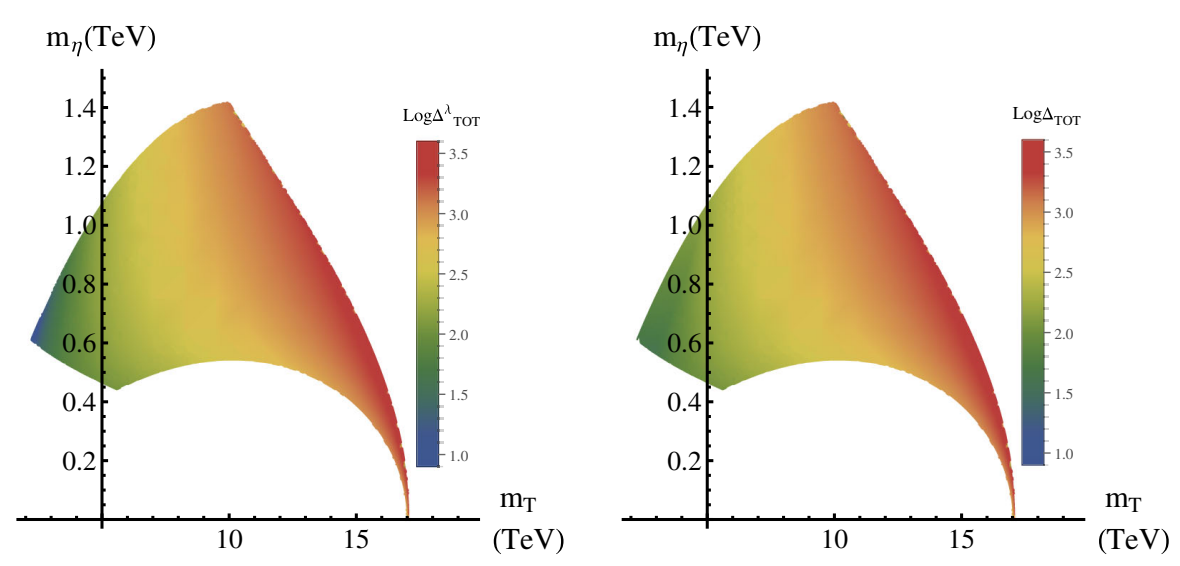

FIG. 6. Left: Density plot of $\log \Delta_{\mathrm{TOT}}^{\lambda}$ in the $m_{\eta}-m_{T}$ plane for $f=10 \mathrm{TeV}$. Right: Density plot of $\log \Delta_{\mathrm{TOT}}$ in the $m_{\eta}-m_{T}$ plane for $f=10 \mathrm{TeV}$. In both plots Log means $\log _{10}$.
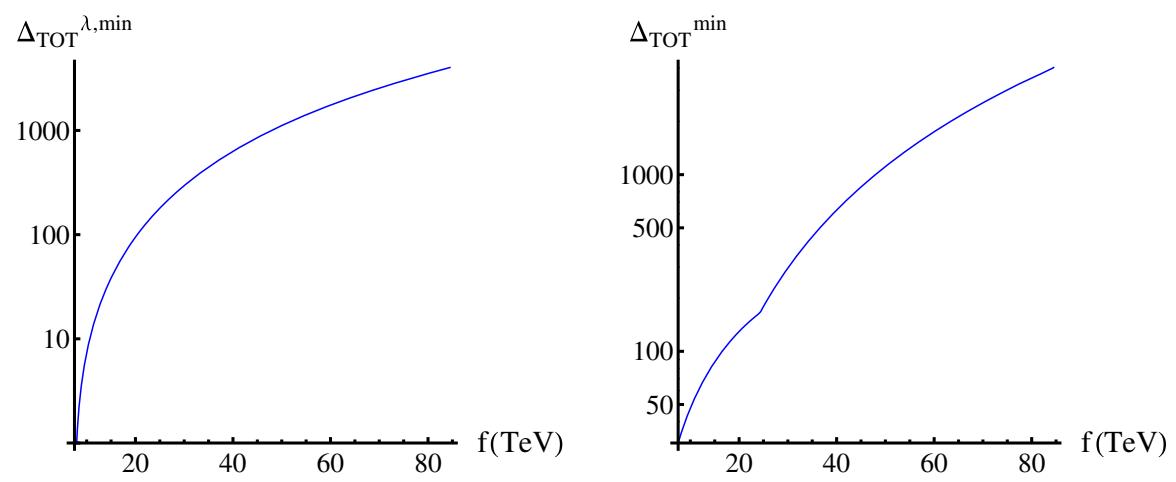

FIG. 7. Left: Minimum $\Delta_{\text {TOT }}^{\lambda}$ as a function of $f$. Right: Minimum $\Delta_{\text {TOT }}$ as a function of $f$.

(near the unitarity boundary), with $\Delta_{\mathrm{TOT}}^{-1}$ approaching a few percent. The favored pseudoaxion mass is around $600 \mathrm{GeV}$ in this case. Unfortunately the parameter region corresponding to a light pseduoaxion with mass smaller than $400 \mathrm{GeV}$ is strongly disfavored by naturalness, as can be inferred from the figure. Our results shown here to some extent answers from the CEFT point of view the question in Ref. [40] which raises concern about the fine-tuning in the SLH given a strong constraint on $f$.

Figure 7 shows the minimum allowed $\Delta_{\mathrm{TOT}}^{\lambda}$ and $\Delta_{\mathrm{TOT}}$ as a function of $f$. It is not surprising that both of them exhibit a monotonically increasing behavior. We note that for a sufficiently small $f \sim 7.5 \mathrm{TeV}, \Delta_{\text {TOT }}^{\lambda}$ can reach zero, indicating that a vanishing $\lambda_{U}$ at the unitarity cutoff is still allowed. However this value of $f$ is already near the boundary of LHC exclusion limit. For larger $f, \Delta_{\text {TOT }}^{\lambda}=$ 0 is then impossible which implies the NDCC assumption with exactly vanishing $\lambda_{U}$ is no longer valid. The value of minimum $\Delta_{\text {TOT }}$ is determined by $\Delta_{\text {TOT }}^{\mu^{2}}$ for $f<25 \mathrm{TeV}$ and $\Delta_{\text {TOT }}^{\lambda}$ for larger $f$. This yields a slight kink at $f \sim 25 \mathrm{TeV}$ in the right panel of Fig. 7.

\section{DISCUSSION AND CONCLUSIONS}

In this work we have analyzed the SLH scalar potential in an approach consistent with the spirit of CEFT. The most important message we obtained from the analysis is a mass relation connecting the pseudoaxion mass and the top partner mass, Eq. (59). The anticorrelation between these two masses gives rise to interesting constraints on the SLH parameter space. Especially, the minimally allowed $t_{\beta}$ increases with $f$ for $f>18 \mathrm{TeV}$ (Fig. 1). On the other hand, the unitarity constraint leads to a maximally allowed $t_{\beta}$ independent of $f$. Therefore an absolute upper bound on $f(f<85 \mathrm{TeV})$ is obtained. This in turn implies absolute upper bound on $Z^{\prime}$ mass $m_{Z^{\prime}}<48 \mathrm{TeV}$ and top partner mass $m_{T}<19 \mathrm{TeV}$. Pseudoaxion mass is also bounded from above: $m_{\eta}$ can reach the maximum allowed value of $m_{\eta} \approx 1.5 \mathrm{TeV}$ when $f \approx 51 \mathrm{TeV}$. We emphasized the importance of retaining the field dependence in the field form factor $\Delta(\hat{h})$ for a quantitative analysis of the scalar potential, which is often ignored by previous studies.

We have also analyzed the issue of Higgs mass naturalness in the SLH in an approach consistent with the spirit 
of CEFT. The parameter space with the least fine-tuning turns out to be characterized by a low value of $f$ and a small $m_{T}$, with an inverse total tuning $\Delta_{\text {TOT }}^{-1}$ at a few percent level. This can be achieved if $t_{\beta}$ is relatively large, close to the unitarity upper bound, while predicting a pseudoaxion mass at around $600 \mathrm{GeV}$.

Although the pseudoaxion mass is bounded from above by a not-very-large value of about $1.5 \mathrm{TeV}$, it is nevertheless quite challenging to detect such a particle at current and future colliders. The reason is that the pseudoaxion couplings to SM particles are all suppressed by $\frac{v}{f}$, and in some cases even by $\frac{v^{3}}{f^{3}}$ (such as the antisymmetric $\mathrm{ZH} \eta$ vertex [26]). Considering the current bound on $f$ $(f>7.5 \mathrm{TeV})$ the suppression is already quite significant. In such a situation it is helpful to consider detecting $\eta$ from the decay of other new heavy resonances such as $Z^{\prime}, T$ [13,41], other heavy quarks or leptons. The $Z^{\prime}$ search using dilepton channel is expected to be most promising in terms of discovery or most stringent in terms of constraint. Taking into account off-shell $Z^{\prime}$ contributions [42] may even extend the reach and be helpful to cover the very heavy region at a $100 \mathrm{TeV} p p$ collider. Another important target for future collider search should be the top partner. Although the heaviest mass value of about $19 \mathrm{TeV}$ is beyond the reach of even a $100 \mathrm{TeV} p p$ collider, the parameter region favored by naturalness consideration should be well in reach.

A full program of testing the mass relation Eq. (59) is conceivable if the associated mass scales are in reach of proposed future colliders. This should be the case for a $100 \mathrm{TeV} p p$ collider if the SLH is realized in nature with $\Delta_{\mathrm{TOT}}^{-1}$ at a few percent level. Four crucial quantities need to be measured, namely $f, t_{\beta}, m_{\eta}$, and $m_{T} . f$ can be determined once the $Z^{\prime}$ is discovered and its mass measured at, for example, the dilepton channel. For the region with small fine-tuning, the top partner should be relatively light and therefore be within reach in the pair production channel. The pseudoaxion $\eta$ might be discovered via $Z^{\prime}$ or top partner decay. Finally, some couplings related to the top partner and other heavy fermions are related to $t_{\beta}[34]$ and might provide a measurement thereof. Combining all the information it would then be possible to understand or veto the EWSB as the consequence of perturbative vacuum misalignment during the global symmetry breaking Eq. (1).

It is certainly warranted to make a comparison between our CEFT-based approach to analyze the scalar potential and the approach based on the NDCC assumption. An even sharper question might be: what is the virtue of the CEFTbased approach compared to using a "floating cutoff" in previous analyses? We may criticize an analysis using the
NDCC assumption with a fixed cutoff value by noting that any of its EWSB predictions which depend on the choice of the cutoff have an uncertainty which is hard to quantify. However, if a floating cutoff is used instead of a fixed value, then it seems that it would yield the same parameter space as compared to our CEFT-based approach. Our answer to this question consists of two aspects. First, it is always desirable that physics theory be not only a tool for calculation, but also can be interpreted in a conceptually consistent manner. In this regard, the practice of imparting physical meaning to the regularization cutoff is not conceptually solid. For example, if we consider an asymptotically free theory like QCD, then we would realize that its perturbative calculation involves logarithmic divergences which need not be cut off by any new particles since QCD can be consistently extrapolated to arbitrarily high energy, yet the divergences are actually harmless [18]. Another example is that imparting physical meaning to the regularization cutoff could lead to violation of field redefinition invariance, as pointed out in Ref. [22]. On the other hand, the CEFT-based approach is based on standard renormalization theory and is therefore conceptually clear. Second, from a practical point of view, the CEFT approach makes manifest the reliable prediction of the theory. In the SLH case, it is the mass relation which has been hidden for a long time under previous studies. It is also worth noting that the CEFT-based approach facilitates the analysis of physical fine-tuning, as demonstrated in Sec. IV.

The CEFT-based approach to scalar potential and finetuning analysis adopted in this work can be carried over to a wide class of little Higgs and twin Higgs models as well. The expected outcome should contain at least various mass relations which pertain to each of the model under investigation. These mass relations should serve as crucial tests of the related EWSB mechanism. The parameter space with small fine-tuning is expected to be associated with a small top partner mass. In conjunction with other theoretical considerations (e.g., perturbative unitarity and vacuum stability) and experimental probes, it is hopeful that we may obtain a deeper understanding of the EWSB, one of the most important pillars of contemporary particle physics.

\section{ACKNOWLEDGMENTS}

We thank Hsin-Chia Cheng and Wai-Yee Keung for helpful discussion. This work was supported in part by the Natural Science Foundation of China (Grants No. 11135003, No. 11375014 and No. 11635001), the China Postdoctoral Science Foundation (Grant No. 2017M610992) and the MoST of Taiwan under the Grant No. 105-2112-M-007-028-MY3. 
[1] G. Aad et al. (ATLAS), Phys. Lett. B 716, 1 (2012).

[2] S. Chatrchyan et al. (CMS), Phys. Lett. B 716, 30 (2012).

[3] G. F. Giudice, Proc. Sci., EPS-HEP2013 (2013) 163 [arXiv:1307.7879].

[4] G. F. Giudice, arXiv:0801.2562.

[5] N. Arkani-Hamed, A. G. Cohen, and H. Georgi, Phys. Lett. B 513, 232 (2001).

[6] N. Arkani-Hamed, A. G. Cohen, T. Gregoire, and J. G. Wacker, J. High Energy Phys. 08 (2002) 020.

[7] N. Arkani-Hamed, A. G. Cohen, E. Katz, E. Katz, T. Gregoire, and J. G. Wacker, J. High Energy Phys. 08 (2002) 021.

[8] N. Arkani-Hamed, A. G. Cohen, E. Katz, and A. E. Nelson, J. High Energy Phys. 07 (2002) 034.

[9] M. Schmaltz and D. Tucker-Smith, Annu. Rev. Nucl. Part. Sci. 55, 229 (2005).

[10] M. Perelstein, Prog. Part. Nucl. Phys. 58, 247 (2007).

[11] D. E. Kaplan and M. Schmaltz, J. High Energy Phys. 10 (2003) 039.

[12] M. Schmaltz, J. High Energy Phys. 08 (2004) 056.

[13] W. Kilian, D. Rainwater, and J. Reuter, Phys. Rev. D 71, 015008 (2005).

[14] W. Kilian, D. Rainwater, and J. Reuter, Phys. Rev. D 74, 095003 (2006); 74, 099905(E) (2006).

[15] J. Reuter and M. Tonini, J. High Energy Phys. 02 (2013) 077.

[16] A. Manohar and H. Georgi, Nucl. Phys. B234, 189 (1984).

[17] X.-F. Han, L. Wang, J. M. Yang, and J. Zhu, Phys. Rev. D 87, 055004 (2013).

[18] M. E. Peskin and D. V. Schroeder, An Introduction to Quantum Field Theory (Addison-Wesley, Reading, MA, 1995).

[19] S. R. Coleman and E. J. Weinberg, Phys. Rev. D 7, 1888 (1973).

[20] J. A. Casas, J. R. Espinosa, and I. Hidalgo, J. High Energy Phys. 03 (2005) 038.
[21] Z. Chacko, H.-S. Goh, and R. Harnik, Phys. Rev. Lett. 96, 231802 (2006).

[22] C. P. Burgess and D. London, Phys. Rev. D 48, 4337 (1993).

[23] S. Chang and H.-J. He, Phys. Lett. B 586, 95 (2004).

[24] H. Georgi, Annu. Rev. Nucl. Part. Sci. 43, 209 (1993).

[25] T. Appelquist and J. Carazzone, Phys. Rev. D 11, 2856 (1975).

[26] S.-P. He, Y.-n. Mao, C. Zhang, and S.-h. Zhu, Phys. Rev. D 97, 075005 (2018).

[27] Y.-n. Mao, Phys. Rev. D 97, 075031 (2018).

[28] F. del Aguila, J. I. Illana, and M. D. Jenkins, J. High Energy Phys. 03 (2011) 080.

[29] O. C. W. Kong, J. Korean Phys. Soc. 45, S404 (2004).

[30] O. C. W. Kong, Phys. Rev. D 70, 075021 (2004).

[31] W. Siegel, Phys. Lett. 84B, 193 (1979).

[32] M. Quiros, in Proceedings, Summer School in High-energy physics and cosmology: Trieste, Italy, 1998 (1999), p. 187, arXiv:hep-ph/9901312.

[33] K. Cheung and J. Song, Phys. Rev. D 76, 035007 (2007).

[34] T. Han, H. E. Logan, and L.-T. Wang, J. High Energy Phys. 01 (2006) 099.

[35] M. Aaboud et al. (ATLAS), J. High Energy Phys. 10 (2017) 182.

[36] R. Barbieri, Phys. Scripta T158, 014006 (2013).

[37] B. Grinstein, R. Kelley, and P. Uttayarat, J. High Energy Phys. 09 (2009) 040.

[38] R. Barbieri and G. F. Giudice, Nucl. Phys. B306, 63 (1988).

[39] B. Yang, G. Mi, and N. Liu, J. High Energy Phys. 10 (2014) 047.

[40] A. G. Dias, C. A. de S. Pires, and P. S. Rodrigues da Silva, Phys. Rev. D 77, 055001 (2008).

[41] K. Cheung, J. Song, P. Tseng, and Q.-S. Yan, Phys. Rev. D 78, 055015 (2008).

[42] S. Alioli, M. Farina, D. Pappadopulo, and J. T. Ruderman, Phys. Rev. Lett. 120, 101801 (2018). 